\title{
Una concepción del tiempo no-lineal en dos lenguas: el maya yucateco colonial y actual y la lengua de señas maya yucateca
}

A conception of nonlinear time in two languages: colonial and present-day

Yucatec Maya, and Yucatec Mayan Sign Language

Une conception du temps non linéaire dans deux langues : le maya yucatèque

colonial et actuel et la langue des signes maya yucatèque

Olivier Le Guen

\section{OpenEdition}

\section{Journals}

Edición electrónica

URL: http://journals.openedition.org/jsa/15327

DOI: 10.4000/jsa.15327

ISSN: $1957-7842$

Editor

Société des américanistes

Referencia electrónica

Olivier Le Guen, « Una concepción del tiempo no-lineal en dos lenguas: el maya yucateco colonial y actual y la lengua de señas maya yucateca », Journal de la société des américanistes [En línea], Maya times | 2017, Publicado el 31 diciembre 2017, consultado el 30 abril 2019. URL : http:// journals.openedition.org/jsa/15327 ; DOI : 10.4000/jsa.15327 


\title{
Una concepción del tiempo no-lineal en dos lenguas: el maya yucateco colonial y actual y la lengua de señas maya yucateca
}

\author{
Olivier LE GUEN*
}

\begin{abstract}
En este trabajo se plantea la existencia una concepción del tiempo propiamente maya (yucateca) en la cual el tiempo no es metafóricamente lineal y puede ser concebido como "estructuralmente cíclico". Usaré ejemplos de dos lenguas que podemos considerar como pertenecientes a una misma cultura maya yucateca. La primera es el maya yucateco y se considera en una perspectiva diacrónica, tomando ejemplos del maya actual pero también del maya colonial, en particular extractos de varios textos del Chilam Balam. La segunda lengua es la lengua de señas maya yucateca; una nueva lengua que surgió en pueblos mayas en los cuales nacieron personas sordas. Para comunicarse sin la ayuda del canal oral, los sordos juntos con sus familiares inventaron un lenguaje señado que tiene características similares en diferentes pueblos. Este trabajo se enfoca en el análisis de las metáforas que se usan para hablar del tiempo y se argumenta que no hubo una influencia de la concepción lineal occidental del tiempo (por lo menos hasta muy recientemente) en la concepción maya del tiempo. [Palabras claves: tiempo, metáforas, maya yucateco, lengua de señas maya yucateca, maya colonial, gestos, Chilam Balam.]
\end{abstract}

A conception of nonlinear time in two languages: colonial and present-day Yucatec Maya, and Yucatec Mayan Sign Language. This work shows to what extent there exists a distinctive (Yucatec) Maya conception of time, in which time is not metaphorically linear and can be conceived as "structurally cyclical." I will take examples from two languages that can be considered to belong to the same culture, namely Yucatec Maya culture. The first is Yucatec Maya considered from a diachronic perspective, with examples from contemporary Maya, as well as examples of colonial Maya taken from the Books of Chilam Balam. The second language considered is Yucatec Mayan Sign Language, an emerging language that recently appeared in Mayan villages where deaf persons were born. In order to communicate without using an oral language, deaf persons and their families have invented sign languages that have similar characteristics in different villages. This work specifically analyses the metaphors used to talk about time and aims to demonstrate that, at least until recently, the western conception of time has not had an influence on the Maya.

* Centro de Investigaciones y Estudios Superiores en Antropología Social, México [ompleguen@gmail.com]. 
[Key words: time, metaphors, Yucatec Maya, Yucatec Mayan Sign Language, colonial Maya, gestures, Chilam Balam.]

Une conception du temps non linéaire dans deux langues : le maya yucatèque colonial et actuel et la langue des signes maya yucatèque. Ce travail montre dans quelle mesure il existe une conception du temps spécifiquement maya (yucatèque) dans laquelle le temps n'est pas métaphoriquement linéaire et peut être conçu comme « structurellement cyclique ». J'utiliserai des exemples de deux langues que l'on peut considérer comme appartenant à une même culture, la culture maya yucatèque. La première langue est le maya yucatèque ici considéré dans une perspective diachronique, avec des exemples du maya actuel autant que du maya colonial, en particulier des textes du Chilam Balam. La seconde langue est la langue des signes maya yucatèque, une langue émergente apparue récemment dans des villages mayas où sont nés des personnes sourdes. Pour communiquer sans l'aide de la langue orale, les sourds avec l'aide de leur famille ont inventé des langages signés qui ont des caractéristiques similaires dans différents villages. Ce travail analyse de façon précise les métaphores utilisées pour parler du temps et entend démontrer qu'il n’y a pas eu (au moins jusqu'à récemment) d'influence de la conception occidentale du temps chez les Mayas. [Mots-clés : temps, métaphores, maya yucatèque, langue des signes maya yucatèque, maya colonial, gestes, Chilam Balam.]

\section{Introducción}

En este artículo se demuestra la existencia de una concepción del tiempo propiamente maya yucateca en la cual el tiempo no es metafóricamente lineal y puede ser concebido como "estructuralmente cíclico". Usaré ejemplos de dos lenguas que podemos considerar como pertenecientes a una misma cultura maya yucateca. La primera es el maya yucateco y se considera desde una perspectiva diacrónica, tomando ejemplos del maya actual y del maya colonial, a través de extractos de textos del Chilam Balam. La segunda lengua es la lengua de señas maya yucateca, que es una nueva lengua que surgió en pueblos mayas en los cuales se encuentran personas sordas. En particular, se consideran las metáforas usadas para hablar del tiempo que nos permiten entender cómo se representa conceptualmente entre los mayas. Los datos analizados tienden a demostrar que no hubo una influencia de la concepción occidental lineal del tiempo (por lo menos hasta recientemente) y que tanto en la lengua maya colonial como en la actual y en la lengua de señas maya yucateca, no existe una concepción lineal sino cíclica del tiempo.

El artículo pretende demostrar dos aspectos diferentes respecto a la concepción del tiempo maya que no son exclusivos. En primer lugar, se sostiene que no existe una metáfora del tiempo lineal en maya yucateco actual o colonial ni tampoco en la lengua de señas maya yucateca. Esta demostración se hace evidenciando la ausencia de metáforas lingüísticas que indican una línea o un eje temporal con direccionalidad (p. ej. frente-atrás, abajo-arriba, etc.). Por 
otra parte, se muestra que sí existen metáforas que apuntan a una concepción del tiempo de tipo cíclica (es decir que el tiempo "da vuelta", puede "ir" y "regresar" sin dirección específica): en la concepción maya, los eventos se remplazan (por lo menos metafóricamente) uno con el otro. Esta concepción cíclica se puede considerar como "estructural" en el sentido en que no es que se repiten los mismos eventos, sino más bien que existe un marco conceptual y organizacional en el cual los eventos re-ocurren con una forma y una valencia similar (esto se muestra en especial con los datos coloniales del Chilam Balam).

La introducción considera las metáforas temporales en varias lenguas del mundo tanto a nivel lingüístico como gestual, la concepción del tiempo tal como se ha considerado en la literatura sobre los mayas; asimismo se presentan las comunidades de estudio y las fuentes históricas coloniales. Una segunda sección examina las expresiones del tiempo en maya contemporáneo, analizando las metáforas del tiempo y sus expresiones gestuales. La tercera sección considera cómo la concepción cíclica y no lineal también está presente en una nueva lengua de señas, la lengua de señas maya yucateca. Finalmente, la cuarta sección retoma como marco de análisis las metáforas actuales del tiempo para explorar las metáforas del tiempo en la profecía hun ajaw presente en varios textos del Chilam Balam.

Esta organización se justifica por el hecho de que este trabajo se basa en publicaciones anteriores donde se muestra más detalladamente la ausencia de una línea temporal metafórica en el maya yucateco actual así como en la lengua de señas maya yucateca y que nos conduce a argumentar por una representación cíclica del tiempo en maya (Le Guen 2012; Le Guen y Pool Balam 2012). La aportación de este artículo radica en su perspectiva comparativa, mostrando cómo las concepciones no-lineal y cíclica existen en las dos lenguas actuales $\mathrm{y}$, cómo las podemos rastrear en textos coloniales a pesar de que son fuentes limitadas y de que se trata de un género específico.

\section{Las metáforas temporales en las lenguas del mundo}

El tiempo es una noción abstracta difícil de representar por sí misma. Por eso, en muchas lenguas del mundo (aunque no en todas, véase Sinha, da Silva Sinha, Zinken y Sampaio 2011), el tiempo se ve modelado y conceptualizado con referencia al ámbito espacial ${ }^{1}$. En este sentido, el lenguaje juega un papel decisivo para determinar de qué manera el tiempo está representado conceptualmente, o "espacializado". Sin embargo, aun cuando el tiempo se expresa a través del dominio espacial, existen diferencias culturales en las formas en las que se concretiza.

1. Estas metáforas son llamadas "conceptuales" por Lakoff y Johnson (1980), es decir que operan eligiendo un concepto concreto o físico que actúa como fuente, y sirve para el entendimiento de un concepto más abstracto o "meta". 
En la mayoría de los idiomas indoeuropeos existe una línea conceptual del tiempo que se desarrolla desde el punto de vista del locutor como sigue: el futuro está en frente del hablante y el pasado se ubica detrás del hablante. Esta representación conceptual está reflejada (y al mismo tiempo sostenida) en el discurso, en particular a través del uso de términos espaciales para hablar del tiempo. Por ejemplo, en francés se considera que uno tiene "la vida ante sí" (avoir la vie devant soi) y "poner el pasado hacia atrás" (laisser/avoir le passé derrière soi). En este sentido es una metáfora productiva (Lakoff y Johnson 1980) que se basa en una línea conceptual del tiempo culturalmente determinada ${ }^{2}$.

En español se nota una transferencia del uso de términos espaciales para hablar del tiempo, así como se presenta en la Tabla 1.

\begin{tabular}{|l|l|}
\hline \multicolumn{1}{|c|}{ Espacio } & \multicolumn{1}{c|}{ Tiempo } \\
\hline Las faldas se vuelven más cortas & Los días se vuelven más cortos \\
Es un largo camino & Fue un largo día \\
La próxima persona en la cola & El próximo domingo \\
Se divide el cuarto en dos & El año se divide en 365 días \\
Desde México hasta Puebla & Desde el lunes hasta el viernes \\
\hline
\end{tabular}

Tabla 1. Ejemplos de transferencia del uso de términos espaciales para hablar del tiempo en español.

La metáfora lineal del tiempo se encuentra en otras culturas, pero a veces a la inversa, así como en aymara, un idioma hablado en los Andes (en Bolivia, Perú y Chile). Núñez y Sweetser (2006) muestran que la metáfora del tiempo en aymara está basada en la idea de que el pasado es "el tiempo del ojo" considerando que lo que pasó ya es visible (ej. 1), mientras que el futuro queda detrás de uno, como "el tiempo de espalda" porque no es visible ni tampoco conocido (ej. 2) (ejemplos en Núñez y Sweetser 2006, p. 415).
(1) Nayra
pacha/timpu u $^{3}$
ojo/visión/frente
tiempo
"el tiempo pasado"
(2) Qhipa
espalda/atrás
"el tiempo futuro"

\author{
pacha/timpu \\ tiempo
}

2. Para más detalles sobre las otras formas en las que el tiempo puede ser espacializado en lenguas europeas, véase Calbris 1990; Casasanto, Fotakopoulou y Boroditsky 2010; Casasanto y Jasmin 2012; Casasanto y Boroditsky 2008; Cooperrider y Núñez 2009.

3. Timpu del español, "tiempo". 
Tanto los ejemplos de las lenguas indoeuropeas como los del aymara consideran una línea temporal horizontal pero en otros idiomas encontramos una línea conceptual vertical, así como en el caso del chino mandarín (aun si existe también una línea horizontal) (Boroditsky 2000, 2001). Por ejemplo, se considera que el mes próximo es el "mes de abajo" cuando el mes pasado es el "mes de arriba". Estos ejemplos implican un contraste entre pasado y futuro que también empieza desde un punto deíctico ubicado en el cuerpo del hablante.

Tanto en las lenguas indoeuropeas como en aymara o en chino mandarín el punto de vista del hablante es determinante para proyectar el tiempo de manera lineal. Si consideramos la proyección del tiempo respecto a los marcos de referencia, se puede decir que el tiempo se proyecta desde un marco de referencia egocéntrico (Bender, Rothe-Wulf, Hüther y Beller 2012).

Sin embargo, no todos los idiomas consideran el punto de vista del hablante y la proyección del espacio al tiempo se puede hacer respecto a un marco de referencia geocéntrico. En este caso la proyección conceptual del tiempo se basa en una orientación estable tomando, por ejemplo, las referencias extracorporales como los puntos cardinales. En kuuk thaayorre (un idioma del norte de Australia) los hablantes usan nombres de puntos cardinales para orientarse espacialmente y metafóricamente para proyectar el tiempo. En esta cultura, el recorrido del sol es la base de una metáfora para el desarrollo del tiempo: el movimiento del sol se extiende metafóricamente al futuro (hacia el poniente) y al pasado (hacia el oriente) y no se limita al día (véase Boroditsky y Gaby 2010; Gaby 2012).

\section{Metáforas temporales en la gestualidad}

Las metáforas que existen en todos las lenguas consideradas anteriormente son productivas y se puede sostener que están ancladas en las representaciones mentales de los hablantes. Existen dos argumentos principales para sostener esta propuesta. El primero es que los hablantes pueden crear nuevas (aun efímeras) metáforas (Lakoff y Johnson 1980). Otro argumento viene de la producción gestual que refleja las metáforas que existen en la lengua. Se nota en particular una adecuación con las metáforas lingüística que se representan visualmente con los gestos (ver Fig. 1).

Es de notar sin embargo que existe una segunda línea temporal horizontal en muchas lenguas europeas que se desarrolla también desde el punto de vista del hablante pero hacia la derecha y hacia la izquierda. En este caso, la proyección de la temporalidad dependerá si la persona es diestra (el pasado queda del lado izquierdo y el futuro del lado derecho) o zurda (en cual caso es el inverso de los diestros). Lo interesante es que esa línea no está sostenida lingüísticamente (es decir, no se habla del futuro estando a la "derecha" o la "izquierda") y existe probablemente por razones de visibilidad, sobre todo cuando se consideran varios eventos secuenciales, ver la discusión en Cooperrider y Núñez (2009). 


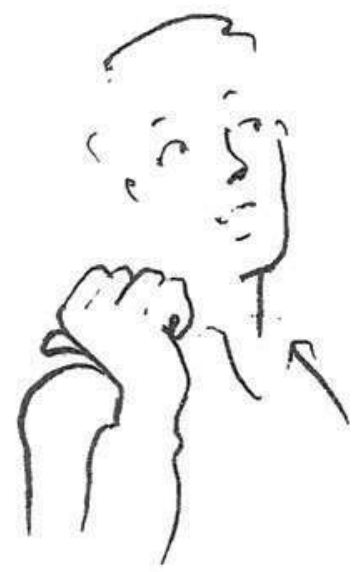

Fig. 1 - Ejemplo de gesto apuntando hacia atrás para hablar del pasado: “en la entrevista que hice en la mañana" (tomado de Calbris 1990, p. 88).

De la misma forma, los hablantes de aymara, tienen una línea gestual que también es horizontal, sin embargo es inversa a la de los hablantes de francés siguiendo el padrón lingüístico que vimos anteriormente. En el estudio conducido por Núñez y Sweetser, los autores muestran cómo los hablantes de aymara (que sólo conocen un poco de español) mientras hablan de eventos pasados tienden a producir gestos orientados hacia delante, así como el la Figura 2.

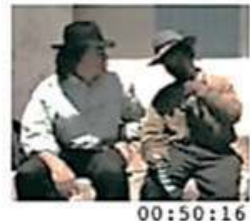

(a)

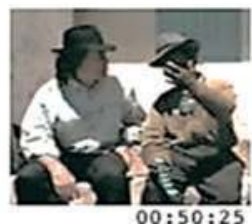

(b)

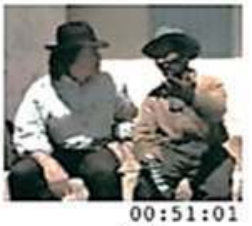

(c)

Fig. 2 - Ejemplo de gesto hacia delante producido en conjunto con la palabra "antiguo" (tomado de Núñez y Sweetser 2006, p. 429).

Entre los hablantes de kuuk thaayorre se usa el recorrido del sol para indicar gestualmente las horas del día, por ejemplo apuntar directamente hacia arriba indica el mediodía y apuntar al poniente refiere a la tarde. Sin embargo, esta misma línea se usa de manera metafórica y el poniente indica el futuro, y el oriente el pasado. De esta forma, Gaby menciona que ha presenciado el hecho de que hablantes apuntaban hacia el oriente para hablar del pasado distante (por ejemplo “40 años antes") (Gaby 2012, p. 3). 
En un experimento, Boroditsky y Gaby pidieron a hablantes de kuuk thaayorre que ordenaran de manera cronológica cuatro cartas con un evento que se desarrolla temporalmente (p. ej. un árbol que crece, un huevo que se abre y del cual sale un pollito, etc.). Los resultados muestran que en su mayoría, los hablantes usan un marco de referencia geocéntrico para proyectar el desarrollo del tiempo y ubican los eventos más antiguos hacia el oriente cuando ponen los eventos más recientes hacia el poniente, así como se puede apreciar en la Figura 3 (Boroditsky y Gaby 2010; Gaby 2012). Este experimento muestra otra vez que la línea temporal no está anclada al cuerpo del hablante sino en puntos estables en el paisaje.

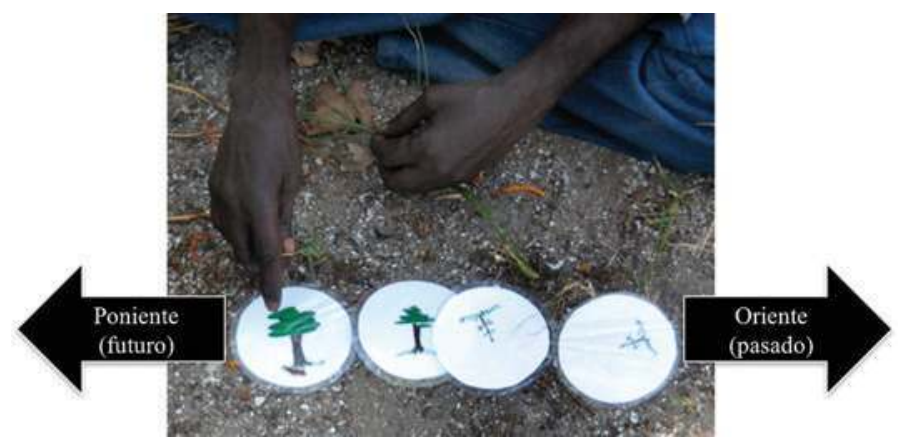

Fig. 3 - Disposición de las cartas según un orden cronológico que se desarrolla del más antiguo (hacia el oriente) al más reciente (hacia el poniente) ${ }^{4}$.

Vimos que, incluso interculturalmente, existe una adecuación entre las metáforas verbales y gestuales. En la siguiente sección, se considera primeramente los estudios clásicos sobre la concepción del tiempo en la literatura maya y se presentan las comunidades de estudio para poder posteriormente explorar las metáforas temporales en las dos lenguas consideradas.

\section{La concepción del tiempo en la literatura maya}

Es importante diferenciar calendario y concepción del tiempo. Mientras que el calendario es una herramienta cultural para calcular intervalos de tiempo, lo que se entiende por concepción del tiempo es la representación conceptual o mental de la forma en la cual se desarrolla el tiempo.

En la literatura mayista en general, y en la que trata de los mayas yucatecos en particular, frecuentemente se menciona la idea de "ciclicidad" del tiempo maya (Bracamonte y Sosa 2010; León-Portilla 2003 inter alia) y muchos datos existen en cuanto al calendario prehispánico, sin embargo, raramente se proporcionan datos 
concretos y convincentes de esta metáfora entre los mayas yucatecos actuales. Es frecuente que los autores se limiten a mencionar el ciclo agrícola o actividades regulares para argumentar una concepción cíclica del tiempo maya. Pero este dato no es suficiente para hablar de una concepción cíclica del tiempo, dado que cualquier sociedad rural o urbana la puede tener. Por ejemplo, el calendario gregoriano que se usa en muchas sociedades occidentales -como México, EE. UU. y Francia, entre otras-incluye una parte cíclica, que es el año de 365 días, y otra lineal: el calendario basado en el año 0 en referencia al nacimiento de Cristo.

Aun cuando hubo una influencia del calendario gregoriano y se adoptó en maya yucateco los nombres españoles de la semana y de los meses, la concepción del tiempo no fue afectada por estos rasgos, como se muestra a continuación. Por ejemplo, no se impuso una línea temporal conceptual, aun cuando otras áreas de la cultura fueron mucho más reorganizadas, como en el caso de la religión.

\section{Comunidades de estudio y fuentes}

Los datos etnográficos provienen de varias comunidades de la península yucateca en los estados de Yucatán y Quintana Roo. En dos comunidades, K'opch'e'en y Che 'ma'ax, se colectaron los datos en maya yucateco y en Chi'kaan y Nohk'op se colectaron datos de la lengua de señas maya yucateca (LSMY). Todos los trabajos de campo fueron conducidos en maya yucateco y en LSMY. Las fuentes coloniales provienen de varios Chilam Balam, de Chumayel, Tizimin y Oxkutzcab.

En el mapa (Fig. 7) se presenta la ubicación geográfica de los pueblos.

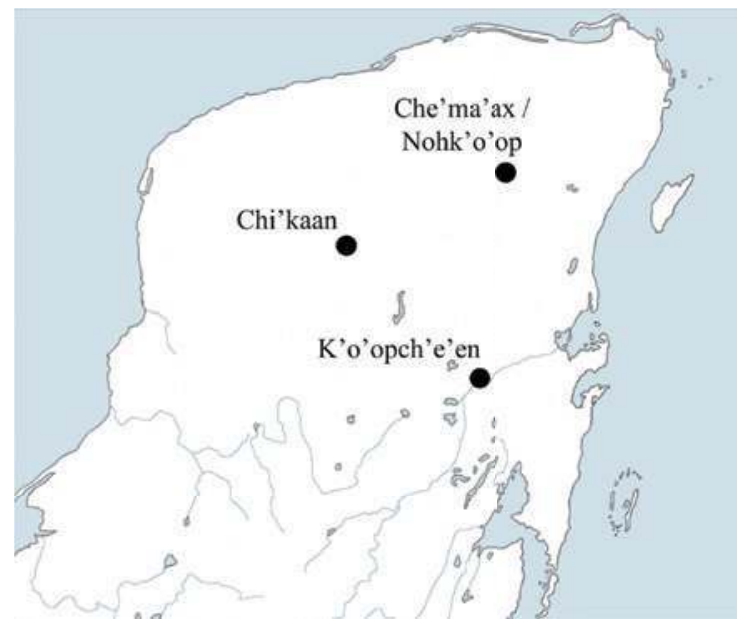

Fig. 4 - Ubicación de los pueblos en los que se han conducido trabajos de campo (*Nohk'o'op está ubicado en el pueblo de Che 'ma'ax). 
A continuación se presenta rasgos de la lengua maya y de la LSMY así como las comunidades de estudio y las fuentes usadas para este análisis.

El contexto maya yucateco y las comunidades de estudio

El idioma maya yucateco (o maaya t'aan, lit. "la lengua/palabra maya") pertenece a la familia lingüística maya, y más específicamente a la rama yucatecana. Es hablado por aproximadamente 800,000 hablantes (INEGI 2010).

El maya yucateco se habla actualmente en toda la península de Yucatán (es decir los estados de Quintana Roo, Yucatán y una parte de Campeche), así como en el norte de Belice. Hasta hace unos 50 años, la cultura maya todavía dependía principalmente de la agricultura de tumba y del cultivo del maíz y de otras plantas como frijoles y cucurbitáceos (Redfield y Villa Rojas 1934; Terán y Rasmussen 1994; Villa Rojas 1987). Sin embargo, la tendencia actual entre los jóvenes, ahora que la escolarización se está reforzando, es optar por trabajos remunerados fuera del pueblo (en las áreas de construcción, restauración, turismo, computación, limpieza, etc.), incluso en el extranjero. Asimismo con la alfabetización en español y el impacto de la televisión en particular, el idioma maya se encuentra en fuerte competencia con el español, incluso dentro del hogar. Sin embargo, la tendencia no es hacia el bilingüismo sino hacia un desplazamiento del maya yucateco por el español.

Aunque existe una forma escrita del idioma (con varios tipos de alfabetos), el maya yucateco es ante todo un idioma hablado. Los hablantes de maya yucateco, cuando quieren escribir, usan el español y leen con dificultad escritos en maya (tales como las biblias o escritos religiosos difundidos por varios grupos evangélicos). Existen algunas radios en la península que difunden programas en maya yucateco. Aunque existen ciertas variaciones dialectales (Blaha Pfeiler 1996, 1999; Blaha Pfeiler y Hofling 2006), éstas son comprensibles, lo que le da al maya yucateco su carácter homogéneo. Por el contrario, las situaciones sociolingüísticas y la vitalidad del idioma varían mucho dentro de la península.

En términos de socialización, la tendencia a la neolocalización (es decir que la nueva pareja se va a vivir a un asentamiento separado) es bastante drástica porque los abuelos, que siempre cumplieron un papel importante y específico -el de la socialización de los niños-, ya no están presentes. En la actualidad, el rol de los abuelos (generalmente monolingües en maya) es particularmente importante en el mantenimiento del maya yucateco, sobre todo con la tendencia de los padres a socializar a sus niños en español. Este cambio lingüístico tiene consecuencias no sólo en el aspecto lingüístico sino también a nivel cognitivo e influye en concepciones como la del tiempo por ejemplo. Sin embargo, el trabajo de investigación de la influencia de la socialización en español sobre las representaciones mentales queda por llevar a cabo. 
Los datos presentados en este trabajo provienen de dos comunidades de habla maya yucateco: Kopchen y Chemax.

Kopchen (o K'opch 'e'en en maya, lit. "el hueco-pozo") es un pueblo ubicado en la zona maya de Quintana Roo, en el municipio de Felipe Carrillo puerto. La población es de aproximadamente 500 habitantes (INEGI 2010) aunque muchos hombres y ahora mujeres, que van a trabajar a la costa (entre Tulum y Cancún) o que estudian en ciudades cercanas (Felipe Carrillo puerto o José María Morelos), regresan solamente los fines de semana. En el pueblo, todos los habitantes hablan maya de forma cotidiana. Los hombres (y las mujeres cada vez más) van aprendiendo un poco de español que usan con los que no pueden (o no quieren) hablar en maya, en general personas de la ciudad. Los niños siguen socializados en el idioma maya aunque se nota una incipiente tendencia hacia una socialización en español (en particular durante los primeros 5 años de vida).

Chemax (o Che'ma'ax en maya, lit. "el árbol del mono araña") es un pueblo colonial ubicado en el municipio epónimo, ubicado en el oriente del estado de Yucatán cerca de la frontera con el estado de Quintana Roo. Tiene una población de aproximadamente 18,000 habitantes $(30,000$ habitantes en todo el municipio). Por razones históricas se habla español y maya. En el centro en particular residen muchos habitantes ladinos de descendencia española (o cuando menos no-indígena) y el resto de la población se distribuye en el centro y en el resto del pueblo. En esta comunidad, se nota una fuerte influencia del español entre la población maya y muchos de los jóvenes son bilingües aunque también se nota la preferencia por un monolingüismo en español. Con la construcción de Cancún en los años setenta y el desarrollo de la zona turística de la costa de Quintana Roo, gran parte de los hombres del pueblo abandonaron la milpa y se fueron a trabajar en la construcción. Resulta que actualmente existe poca actividad agrícola en Chemax y las actividades laborales son muy diversas (Brown 1981, 1994).

\section{La lengua de señas maya yucateca}

La lengua de señas maya yucateca se define como un lenguaje señado que se desarrolla en una comunidad de habla maya yucateca. Es decir que emerge de manera espontánea en varias comunidades mayas yucatecas donde nacen personas sordas. Este contexto particular implica que los señantes de LSMY comparten un fondo cultural con los hablantes de maya yucateco. Por lo mismo, se puede considerar que el maya yucateco y la LSMY son dos idiomas en contacto intenso. En las varias comunidades donde fueron llevadas a cabo encuestas, la mayoría de los señantes ${ }^{5}$ resultó ser hablante de maya yucateco (y

5. El término señante refiere a las personas que se comunican en lengua de señas (que "señan"). Los señantes pueden ser sordos u oyentes. 
a veces también de español). En resumen, la LSMY es creada para el beneficio de una minoría de personas sordas y usada por un gran número de individuos que se relacionan con ellas.

Al considerar sus rasgos sociológicos se puede calificar la LSMY como lengua de señas pueblera. A nivel tipológico, la LSMY es una lengua aislada. Aún está en contacto con el maya yucateco (en particular con los gestos co-verbales usados en esta lengua), sin embargo, es una lengua diferente y no es una versión señada del maya hablado. El conjunto de comunidades de habla estudiadas, en Chicán y Nohkop (respectivamente Chi'kaan y Nojk'o'op en maya) en particular, muestra rasgos lexicales y gramaticales muy semejantes. Sin embargo, queda pendiente un análisis que pueda determinar si se trata de dos lenguas diferentes o si las lenguas de las dos comunidades pueden ser consideradas variantes de una misma lengua de señas maya yucateca.

En términos de contactos con otras lenguas de señas, se tiene que notar una influencia muy periférica de la lengua de señas mexicana (LSM). Se nota en la lengua de señas maya yucateca (o LSMY) de Chicán y de Nohkop algunos préstamos de LSM así como un conocimiento básico del alfabeto manual. Sin embargo, la LSM no se usa en Chicán ni en Nohkop y eso a pesar del esfuerzo de varios grupos (testigos de Jehovah en particular) por introducirla y enseñársela a los sordos.

Por el hecho de que emerge entre comunidades lingüísticas de mayas hablantes, y puesto que estos últimos hacen un uso muy importante de la multimodalidad, no es sorprendente presenciar la transmisión de ciertos gestos (en particular los "gestos citables" o "emblemas") de la gestualidad maya yucateca a la lengua de señas. Como lo vamos a corroborar más adelante, es el caso del tiempo (para más detalles véase Le Guen 2012).

Los datos presentados en este trabajo provienen de dos comunidades en las cuales se usa la LSMY: Chicán y Nohkop.

Chicán (o Chi' Kaan en maya, lit. "la boca de la culebra"), es, hasta ahora, la comunidad documentada con el mayor número de sordos en la península de Yucatán: cuenta con 17 sordos señantes. La comunidad cuenta con unos 720 habitantes, por lo que la proporción de sordos es muy importante (alrededor de un $2.4 \%$ ) (Escobedo Delgado 2012). En esta comunidad, las mujeres de más de 60 años son en general monolingües en maya yucateco mientras que los hombres y algunos miembros más jóvenes de la comunidad son bilingües maya yucateco-español. Sin embargo, las interacciones cotidianas en el pueblo siguen siendo conducidas en maya. El español es enseñado en la escuela y

6. Notar que existe una hipótesis alternativa en cuanto al nombre del pueblo que podría venir, según algunos informantes, de la palabra chikam, "jícama" (la alternancia entre los sonidos $/ \mathrm{n} / \mathrm{y} / \mathrm{m} /$ en fin de palabra es muy frecuente en maya yucateco). 
usado con interlocutores que no hablan maya. La tendencia es socializar a los niños en español, que se habla ahora incluso en la casa. Se puede esperar cambios lingüísticos drásticos dentro de las próximas generaciones. Esta comunidad es muy conocida por su "relativamente" alto número de sordos. La situación lingüística es muy interesante pues la lengua de señas maya que éstos usan también es utilizada por hablantes de maya yucateco de tal forma que en algunos casos algunos son trilingües, ya que hablan maya, lengua de señas y español.

La comunidad de habla de Nohkop? (o Noj K'o'op en maya, lit. "el gran hueco", "la gran caverna") es bastante reducida: se compone de sólo cuatro sordos, todos hermanos, y de unas treinta personas oyentes, hablantes de maya yucateco y de español. La hermandad está compuesta de un total de cinco niños, respectivamente una muchacha de 22 años, otra de 17, un muchacho de 14, una muchacha de 13 y el benjamín, de unos 11 años. La niña de 13 años da muestras de tener un poco de audición y el último niño es oyente. Es interesante notar que la comunidad es mayormente femenina: la familia cuenta con tres niñas (de las cuales dos son las hermanas mayores) así como la prima cuyo papel es también fundamental. Este efecto de género tiene ciertas consecuencias sobre el desarrollo del idioma (como se anticipaba a partir de estudios realizados entre los hablantes de maya yucateco), sobre todo en ciertos campos semánticos tales como el de los colores (se crearon más señas para colores, ocho para ser precisos, en esta comunidad más reducida que en la de Chicán que cuenta con más señantes y que tiene solamente tres términos). Asimismo, aun con una comunidad tan reducida, se notan otras variaciones en la producción de las señas debido al género (genderlect).

\section{Las fuentes: los libros del Chilam Balam}

Para este estudio se consideran tres fuentes que son los Chilam Balam de Chumayel, Oxkuztcab y Tizimin. Los libros de Chilam Balam son documentos maya yucatecos escritos con el alfabeto latino durante la época colonial (entre los siglos XVI y XVIII). La escritura con caracteres latinos se introduce en esa época en los conventos de las ciudades de Campeche, Mérida y Maní y allí se forman los hijos de la nobleza indígena quienes aprenden a leer y escribir el español y el latín así como su propia lengua con dicho sistema de escritura (Cunill 2008). En contraste con muchos otros documentos de la misma época, no tienen fines legales. El contenido de estos textos es mítico, histórico y

7. Nohk'o'op es un nombre inventado que fue usado en lugar del nombre real del pueblo que es Che'ma'ax, para mantener la privacidad de los hablantes y señantes ya que en su mayoría eran menores de edad. Ahora lo usamos para designar el barrio donde se ubica la casa de la abuela de los señantes. 
religioso. En muchos casos, los textos de Chilam Balam son compilaciones de varios textos más antiguos escritos por varios autores.

Desde los principios del siglo xx varias traducciones de los libros de Chilam Balam han sido propuestas. En 1930, Antonio Mediz Bolio propone una traducción del Chilam Balam de Chumayel en español (Mediz Bolio 1930), seguida por Roys (1933, 1967), Edmonson (1986) y Luxton $(1995)^{8}$. El Chilam Balam de Tizimin por su parte, procedente del pueblo de Tizimin (Yucatán), fue descubierto a mitad del siglo XIX. Fue copiado por Berendt posiblemente alrededor de los años 1870. Basándose en esta copia, Brinton propone la primera traducción en 1882 (Brinton 1882), seguida por otra de Maud Worcester Makemson (Makemson 1951). A fines del siglo xx, Edmonson propone su propia interpretación con una paleografía del texto (Edmonson 1982). Finalmente, del Chilam Balam de Oxkutzcab se conocen solamente partes: figuran en el famoso códice Pérez que es una colección de pedazos de textos mayas recopilados por D. Juan Pío Pérez alrededor del año 1940 (Pérez 1949). Se encuentra en este libro la profecía Hun Ahau. Por otra parte, Barrera Vásquez y Rendón han publicado una traducción al español de una complicación de varios fragmentos de los diferentes libros de Chilam Balam encontrados y del Códice Pérez (Barrera Vázquez y Rendón 1948).

\section{Expresión del tiempo y metáforas temporales en maya yucateco contemporáneo}

Antes de explorar las metáforas específicas del tiempo que existen en maya yucateco, es crucial presentar ciertos rasgos gramaticales sobre la expresión de la temporalidad en esta lengua.

\section{La gramática del tiempo}

En maya yucateco, el tiempo (tense) no existe como categoría gramatical (pero véase Vapnarsky 2016). Es decir que no hay, como en español, morfología puramente temporal en los verbos. La información temporal se realiza mediante la combinación de aspectos, modos, adverbios y partículas temporales (para más detalles veáse Bohnemeyer 2002, 2009; Vapnarsky 1999). Consideremos

8. Tres años después de la traducción de Mediz Bolio, Roys produce una versión en inglés del mismo libro (Roys 1933). Roys elabora una nueva edición con una introducción de Thompson en 1967 (Roys 1967). Tal vez la tradución más famosa o más difundida sea la de Munro S. Edmonson que publica una nueva traducción al inglés del Chumayel en 1986 (Edmonson 1986). Finalmente, Luxton publica una nueva traducción del Chumayel al final del siglo xx (Luxton 1995). 
el ejemplo siguiente (3) ${ }^{9}$; para expresar una acción habitual en el pasado, o sea un pretérito imperfecto, el maya yucateco tiene primero que indicar que la oración está ubicada en otra temporalidad (con el adverbio $k a$ 'ach), usar otra forma en participio presente con la raíz suk "acostumbrar" y usar una oración en el incompletivo.
ka'ach-e'
suk-a'an
in-kon-ik
p'aak
meerkado
CTRFACT-TD 4
habituar-PART 1 1A-vender-TR.ICP tomate
mercado
"Vendía tomates en el mercado" [lit. "Antes, estoy acostumbrada a vender tomates en el mercado"]

Asimismo, para expresar un evento en el futuro, el maya tiene varias opciones (véase Vapnarsky 2017, en esta publicación), una de ella siendo el recurso al subjuntivo y a un terminativo, así como se presenta en el ejemplo (4).

$$
\begin{aligned}
& \text { ken káaj-ak (l)e óok'ot-o' ts'ok in-ween-el } \\
& \text { IRR empezar-SBJ DET bailar-TD }{ }_{2} \text { TERM 1A-dormir-PART } 3 \\
& \text { "Ya estaré dormido cuando empiece el baile" [lit. "Cuando empezará el } \\
& \text { baile, termino de estar dormido"] }
\end{aligned}
$$

El maya yucateco, aunque no cuenta con tiempo gramatical recurre a una plétora de adverbios y partículas temporales, así como se presenta en la Tabla 2 y la Tabla 3. Esto contrasta con la dificultad de expresar relaciones temporales que no se relacionan con el evento de habla (ver Le Guen y Pool Balam 2012 para más detalles sobre este punto).

9. ADe aquí en adelante las abreviaciones usadas serán las siguientes: 1: primera persona; 2: secunda persona; 3 : tercera persona; A: marcadores de persona del juego A (ergativo subjeto de verbo transitivo posesor de nominales); AG: agente ; AP: antipasivo; APL: aplicativo; в: marcadores de persona del juego B (absolutivo subjeto de verbos intransitivos de predicados no verbales objeto de verbos transitivos); CAUs: causativo; CONJ: conjunción; CP.INTR: marcador TAM inicial de completivo intransitivo; CTRFACT: contrafactual ( $\left.k a^{\prime} a c h i h\right)$; DEON: deóntico; DEM: demostrativo; DET: determinante; EXST: existencial; FOC: foco; FUT.PROF: futuro profético; HAB: habitual; ICP: incompletivo; INCH: incoativo IRR: irrealis ; MAF: enfoque de manera; NC: clasificador numeral; NOM: nominal; MP: mediopasivo; PART $_{3}$ : participio mediopasivo - $v 1 /$-al; PAS: pasivo; PR: pronombre independiente; PREP: preposición; PROG: progresivo; RECIP: reciproco; REL: relacional -il; SBJ: subjuntivo; SG: singular; TD: deíctico terminal; $\mathrm{TD}_{1}$ : deíctico terminal (proximal/ información nueva); $\mathrm{TD}_{2}$ : deíctico terminal (distal/saber compartido); $\mathrm{TD}_{3}$ : deíctico terminal (locativo/partitivo/negación); $\mathrm{TD}_{4}$ : deíctico terminal (tópico); TERM: terminativo; TR: transitivo; TRZER: transitivizador 


\begin{tabular}{|l|l|}
\hline \multicolumn{1}{|c|}{ Términos mayas } & \multicolumn{1}{c|}{ Equivalente en español } \\
\hline úuch & Tiempo en el pasado distante \\
ka'ach & $\begin{array}{l}\text { Tiempo en el pasado distante (dentro del marco de una vida); } \\
\text { irrealis; marca contrafactual10 }\end{array}$ \\
to'l-ak & Tiempo distante (dentro del marco de unos días) \\
jo'oloj & El día antes \\
sáam(y-ak) & Pasado reciente (dentro del día) \\
táant & Pasado reciente (en términos de minutos) \\
be'oora & Ahora \\
walak(-il-a') & Ahora/al mismo tiempo que ahora (en el pasado o futuro) \\
ta'ayt(-ak) & Futuro inmediato en términos de minutos \\
mun-xáan-tal & Futuro inmediato en términos de horas (dentro del día) \\
mun-(y)úuch tal & Futuro inmediato en términos de días \\
\hline
\end{tabular}

Tabla 2. Adverbios temporales en maya yucateco actual.

\begin{tabular}{|l|l|c|}
\hline \multicolumn{1}{|c|}{$\begin{array}{c}\text { Términos } \\
\text { mayas }\end{array}$} & \multicolumn{1}{|c|}{$\begin{array}{c}\text { Equivalente } \\
\text { en español }\end{array}$} & $\begin{array}{c}\text { Tiempo } \\
\text { a partir de la oración }\end{array}$ \\
\hline óoxyak & "3 días antes" & -3 \\
ka'ajvyak & "2 días antes" & -2 \\
ho'olyak & "ayer" & -1 \\
o’nyajak & "ayer en la noche" & -0.5 \\
bejla('ak)e' & "ahora, en estos días" & 0 \\
sáamal & "mañana" & +1 \\
ka'abeh & "dentro de 2 días" & +2 \\
óoxej & "dentro de 3 días" & +3 \\
\hline
\end{tabular}

Tabla 3. Adverbios temporales refiriendo a distancia entre días en maya yucateco actual.

Finalmente, podemos considerar la construcción que remite a un futuro profético o futuro distal con la partícula temporal bíin y el uso del subjuntivo.

En contraste con el español, el maya no tiene (originalmente) palabas para antes y después (véase Bohnemeyer 2009), lo que obliga a los hablantes a ordenar los eventos de manera cronológica en sus enunciados. En el ejemplo

10. Para más detalles, véase Vapnarsky (1999, cap. 3). 
siguiente (5), para expresar el hecho de que su hija se casó un día antes de que llegará el Santo al pueblo, la hablante tiene que formular los eventos en orden cronológico (ver más adelante). En el caso del ejemplo (5) podemos notar también que la hablante usa una analogía como si el día de la boda fuera hoy y que al día siguiente llegará el Santo.

\begin{tabular}{|c|c|c|c|c|}
\hline $\begin{array}{l}\text { (5) je'ex } \\
\text { DEM }\end{array}$ & $\begin{array}{l}\text { bejlae' } \\
\text { hoy }\end{array}$ & $\begin{array}{l}u \text {-kajtal-e' } \\
3 \mathrm{~A}-\mathrm{casaj}^{-} \mathrm{TD}_{4}\end{array}$ & $\begin{array}{l}\text { ken } \\
\text { IRR }\end{array}$ & $\begin{array}{l}\text { sáas-ak-e } \\
\text { claro- }{ }^{-}{ }^{2}-\mathrm{TD}_{4}\end{array}$ \\
\hline $\begin{array}{l}\text { tun-taal } \\
\text { PROG.3A-venir }\end{array}$ & $\begin{array}{l}\text { le } \\
\text { DET }\end{array}$ & $\begin{array}{l}\text { San } \\
\text { santo }\end{array}$ & $\begin{array}{l}J \\
\text { AGP }\end{array}$ & $\begin{array}{l}\text { Waan-o' } \\
\text { juan- } \mathrm{TD}_{2}\end{array}$ \\
\hline
\end{tabular}

"Es así, digamos que hoy se casara (mi hija) y el día siguiente llegara el San Juan [lit. 'y cuando aclarece otra vez, llega San Juan']” [FKK-NT_02.09.2010]

Este recurso, aunque no único, se usa muy frecuentemente en maya para vincular eventos distantes entre sí. Esta estrategia permite a los hablantes recurrir a una formulación deíctica (preferida en esta lengua) y hacer como si eventos distantes y relacionados entre sí fueran relacionados con el evento de habla.

A pesar de la ausencia de tiempo gramatical en maya yucateco, existen muchos recursos y combinaciones de recursos para expresar las mismas ideas y sucesiones de eventos que en otras lenguas. La sesión siguiente examina otro recurso que es el uso de metáforas.

\section{Metáforas temporales en maya actual}

Aun cuando se atestiguan usos de formas como pachal (de paach "espalda") "después" o táanil (de táan "frente") "enfrente, antes", son muy limitados y no son productivos. Incluso pueden ser contradictorios a primera vista. Comparemos los ejemplos (6), (7), (9) y (8) (para más ejemplos, véase Le Guen y Pool Balam 2012, p. 6-7).

\begin{tabular}{|c|c|c|c|c|c|}
\hline $\begin{array}{l}u-\text { paal-il } \\
\text { 3A-niño-REL } \\
\text { "'mi niñez está }\end{array}$ & $\begin{array}{l}\text { máak-e' } \\
\text { gente--TD } \\
\text { frente a mí }[=\end{array}$ & $\begin{array}{l}\text { táan-il } \\
\text { frente-NOM } \\
\text { antes]" }\end{array}$ & $\begin{array}{l}\text { yaan } \\
\text { EXST }\end{array}$ & $\begin{array}{l}t i^{\prime} \\
\text { PREP }\end{array}$ & $\begin{array}{l}\text { teen } \\
\text { PR.1SG }\end{array}$ \\
\hline $\begin{array}{l}u-\text { nojoch } \\
\text { 3A-grande }\end{array}$ & $\begin{array}{l}\text { máak-il-e' } \\
\text { gente-REL-TD }\end{array}$ & $\begin{array}{l}\text { pach-il } \\
\text { espalda-NOM }\end{array}$ & $\begin{array}{l}\text { yaan } \\
\text { EXST }\end{array}$ & $\begin{array}{l}t i \\
\text { PREP }\end{array}$ & $\begin{array}{l}\text { teen } \\
\text { PR.1SG }\end{array}$ \\
\hline
\end{tabular}

Si bien los usos de táanil en (6) y en (8) a continuación, pueden ser traducidos como "antes" son limitados al tiempo deíctico (es decir que no se puede usar como en español de manera secuencial para relacionar dos eventos que son diferentes del evento de habla). Además, al nivel conceptual tenemos 
una oposición entre el ejemplo (6) en el cual el pasado podría parecer estar enfrente del hablante y el ejemplo (7) en el cual el futuro parece estar ubicado a la espalda del hablante. Basándonos en estos dos ejemplos, se podría inferir que los mayas yucatecos tienen una línea temporal similar a la de los aymara por ejemplo (Núñez y Sweetser 2006). Sin embargo, no es el caso y esto por dos razones. La primera tiene que ver con el hecho de que esta metáfora no es productiva en maya y se limita a unas expresiones fijas como las mencionadas en los ejemplos (6) y (7). En segundo lugar, si el futuro fuera consistentemente ubicado a la espalda del hablante, no podríamos tener el ejemplo (8).
(8) yan u-táan-il-ben-s-a'al u-k'iin $u-k$ 'aaba' DEON 3A-frente-REL-TR-CAUS-PAS 3A-día 3A-nombre "se va a adelantar su cumpleaños (lit. se va a poner más frente el día de su nombre)"

En este último caso si la metáfora fuera coherente con el futuro estando a la espalda deberíamos tener una metáfora tal como "acercar hacia la espalda" y no hacia el frente. Es de notar que sí existe una expresión idiomática con paach-al "retrasado, encontraste después del evento" que no es productiva sino fijada, así como en el ejemplo (9).

(9) jach paach-al-laj-ech

mucho espalda-NOM-INCH-2B

\begin{tabular}{|c|c|c|c|}
\hline tumen & ts'o'ok-ij & $k a^{\prime} \quad j$ & k'uch-ech \\
\hline CONJ & TERM-3B & CONJ CP.INTR & llegar-2в \\
\hline
\end{tabular}

Si bien los usos lineales con táan(-il) “(en) frente” y paach(-il/-al) “(en la) espalda, detrás" no son productivos, existen en maya yucateco muchas referencias temporales y metáforas productivas basadas en la idea de que el tiempo "da vuelta" o "revoluciones". La base de la concepción del tiempo es $k$ 'iin "el sol" que significa por extensión también "día”, es decir una revolución del sol alrededor de la tierra, así como lo conciben los mayas y se extiende a "hora". A partir de esta palabra se derivan muchas expresiones tales como los presentados en el ejemplo (10):

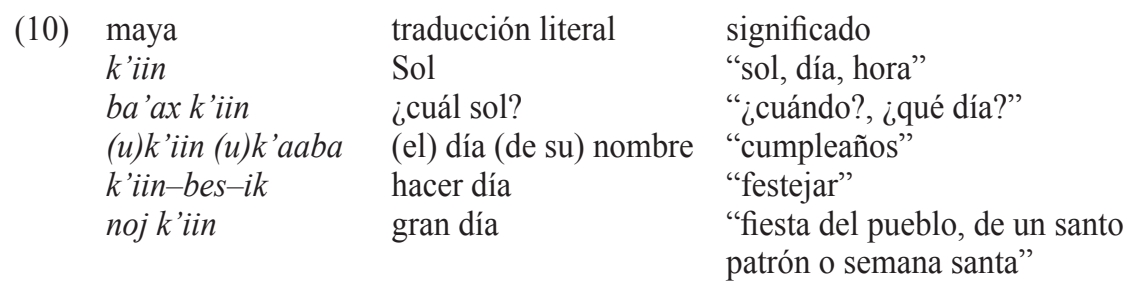


Otra forma de expresar el desarrollo del tiempo, también basada en la idea de ciclo es derivada de la raíz sut, "volver, revolver". Así en maya yucateco jun-súut-uk "un instante" es literalmente "una revolución". El uso de sut es productivo para expresar temporalidad y se integró incluso el préstamo del español sut oora "un instante, de repente" (lit. "la revolución de una/de la hora").

Es de recalcar una vez más que no existe en estas expresiones del tiempo una idea de linealidad (por lo menos que sea productiva) y lo que sobresale, especialmente con la raíz sut "volver, dar vuelta" y de manera inferida con $k$ 'iin "sol, tiempo", es la idea de revolución o ciclicidad del tiempo. Más adelante veremos también metáforas que implican verbos de movimientos.

\section{Metáforas en los gestos co-verbales}

Como se presentó anteriormente, existe una adecuación entre las metáforas lingüísticas y gestuales en varias lenguas, muchas veces basada en la transferencia del dominio espacial al temporal, y así sucede en maya yucateco.

Muchos autores han propuesto la idea que los gestos pueden ser un tipo de "ventana hacia la mente", ya que los gestos permiten expresar de forma visual representaciones internas que a veces no son o no pueden ser expresadas de forma lingüística (de Ruiter 2007; McNeill 1992).

Le Guen y Pool Balam (2012) muestran que no existe una línea metafórica del tiempo en maya yucateco usando varias metodologías y varios tipos de datos: etnográficos, lingüísticos, experimentales y en particular gestuales ${ }^{11}$. El análisis de los gestos muestra que en la producción gestual del maya yucateco no existe una oposición entre pasado y futuro (ver Fig. 7 más abajo); sólo se expresa una oposición "ahora-no ahora" -en contraste con el francés, donde el futuro está en frente y el pasado detrás.

Estos gestos temporales son metafóricos ya que provienen de gestos espaciales que refieren en primer lugar a espacios concretos. Un primer gesto es el de "aquí" usado metafóricamente como "ahora". Figura 5a). Se puede suponer que probablemente es universal ya que es difícil diferenciar el aquí del ahora.

El gesto con la palma abierta encima de la cabeza apunta a un espacio distal o desconocido. Figura 5b). Este gesto no se puede hacer hacia la espalda ya que entre los mayas yucatecos se usa un marco de referencia espacial geocéntrico y apuntar hacia detrás de sí refiere a un espacio real (en realidad esto se extiende a cualquier

11. Para la recolección de los datos del presente artículo, pedimos a cinco hablantes de maya yucateco, que produjeran gestos correspondientes a una lista de conceptos predeterminados (entre otros conceptos temporales), es decir la forma de citación de cada gesto. Preguntamos lo siguiente: bix uye 'esa 'al “¿Cómo se muestra?”: be 'oora/bejlae "“ahora/estos días”, sáamal "mañana", jo'olyak "ayer", ts'uyúuchtal "hace mucho tiempo (pasado)", yan uyúuchtal "dentro de mucho tiempo", sansáamal "todos los días" y kaada áanyo "cada año". Adicionalmente se analizó más de una hora de diversas grabaciones de interacciones espontáneas en cuatro contextos diferentes (véase Le Guen y Pool Balam 2012, p. 9 para más detalles). 
lugar alrededor del hablante). El único lugar que no tiene anclaje concreto se sitúa por lo tanto encima del hablante (en caso que no se trate de apuntar al cielo o un lugar preciso, en cuyo caso generalmente se usará otra forma de mano). El gesto para espacio distante se usa metafóricamente para hablar de un tiempo distante. Lo interesante es que entre los mayas yucatecos el mismo gesto puede ser usado tanto para referir al pasado (generalmente con palabras como úuchij o ts'ok uyúuchtal "hace mucho tiempo (años o más que una vida humana)") como al futuro (con palabras o expresiones como yan uyúuchtal "va a tardar (en términos de años)").

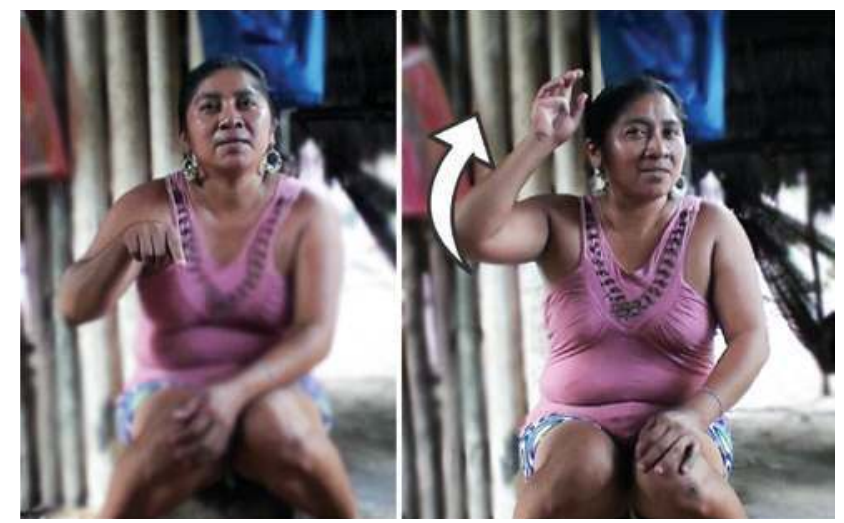

Fig. 5 - Gestos temporales que expresan la oposición “ahora-no ahora" [SCC_ent-conv.gestures_2015.09.15].

En el ejemplo siguiente, un hablante de $K^{\prime}$ 'o 'opch 'e'en está contando al autor que su hija tiene tres años y que va hacia sus cuatro. Explica este proceso formulándolo a través de una metáfora temporal (ejemplo 11).

(11) El hablante explica cuántos años tiene su hija:

$$
\begin{aligned}
& \text { óox-p'ée áanyo yaan ti' be'oora } k-u-b i n \quad t-u \text {-kwaatro áanyos-i' } \\
& \text { tres-NC años EXST PREP ahora HAB-3A-IR PREP-3A-cuatro año-TD } \\
& \begin{array}{llll}
\text { tun-taal } & u \text {-kwaatro } & \text { áanyos } & t-u-\text { paach } \\
\text { PROG.3A-venir } & 3 \mathrm{~A} \text {-cuatro } & \text { año } & \text { PREP-3A-espalda }
\end{array} \\
& \text { ti' } \quad u-t a k^{\prime}-i k \quad \text { ti' juunyo } \\
& \text { PREP 3A-pegar-TR.ICP PREP junio } \\
& \text { "Tiene tres años, ahora va hacia su cuarto año, su cuarto año le viene por la } \\
& \text { espalda/por detrás, le va a pegar (en) junio" [W_2010.11.17] }
\end{aligned}
$$

En este ejemplo, tenemos una oración en tres partes con tres metáforas que parecen contradictorias si nos enfocamos sólo en el habla. En la primera parte, 
el hablante usa una metáfora que considera a la niña como moviéndose hacia su cuarto año, usando el verbo bin "ir". En esta metáfora el tiempo es fijo y es el protagonista (la niña) quien se mueve, en este caso hacia adelante. Sin embargo en la segunda fase, es ahora el tiempo el que se mueve y viene por detrás del protagonista. El hablante nos dice que el tiempo "le viene por detrás". Aquí se nota una contradicción si consideramos las dos metáforas desde la perspectiva del español por ejemplo. ¿Cómo es posible que en un primer tiempo el tiempo sea fijo y que el protagonista se mueva hacia los cuatro años y de repente los cuatro años se muevan hacia la espalda del protagonista? En la tercera fase, tenemos aún otra metáfora en la cual el tiempo no sólo se desplaza según un vector sino que viene a alcanzar al protagonista. El hablante nos dice que los cuatro años "le van a pegar".

Con el fin de entender mejor a qué alude el hablante, el autor le pregunta la siguiente aclaración: bixi? “¿qué?”. El hablante responde lo que se presenta en el ejemplo (12) y en la Figura 6.

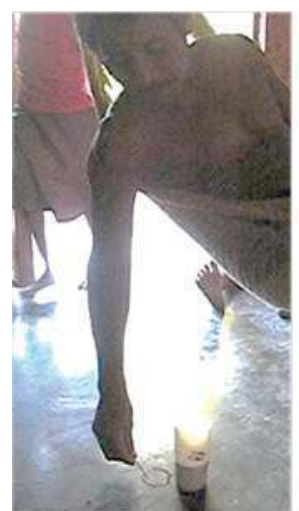

(a)

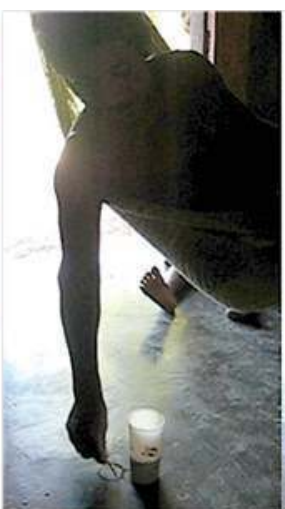

(b)

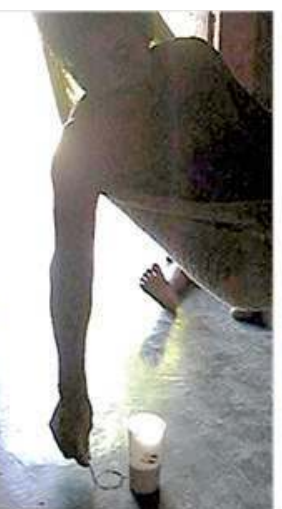

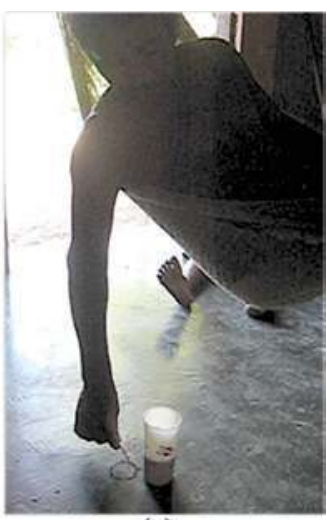

(c)

Fig. 6 - Hablante de maya yucateco que representa visualmente como el tiempo se desarrolla.

(12) explicación de la oración anterior (ej. 11) con gesto (Fig. 6)

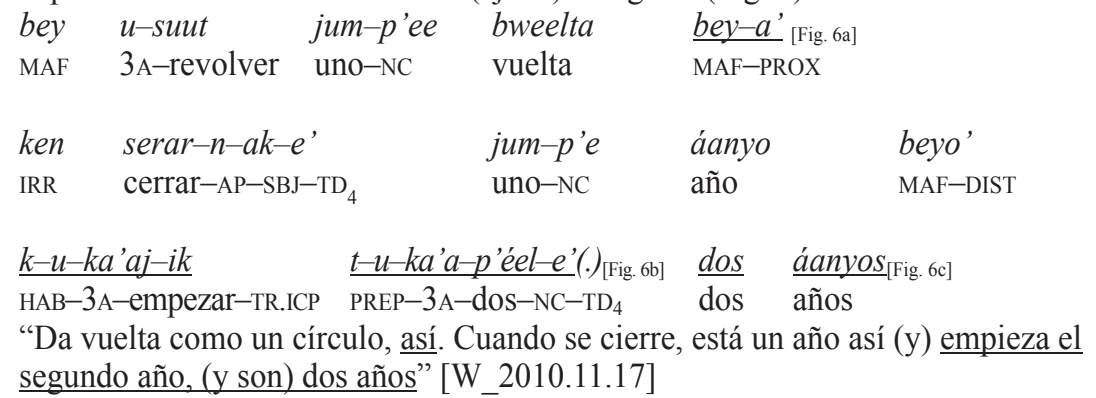


Para explicar cómo se desarrolla el tiempo, el hablante usa de manera ad hoc la marca de agua dejada por un vaso. Este círculo sirve para explicar la trayectoria del tiempo. El primer gesto que hace el hablante va acompañado del deíctico de manera beya" "as's" dibujando un circulo usando la marca de agua (Fig. 6a). Este gesto sirve para ilustrar cómo va el tiempo el primer año (es decir los tres años de edad de la niña). Al cerrar el primer círculo, sigue su movimiento para empezar un segundo (igual que el primero) que ahora acompaña la frase "empieza el segundo año, (y son) dos años" (Fig. 6b,c). Estos círculos son una representación visual de cómo va el tiempo: "revuelve", "da vuelta" y "se cierra". Con esta concepción circular ahora podemos entender el primer enunciado ("la niña va hacia su cuarto año" y "el tiempo le viene por detrás"). La forma no linear del tiempo, sino circular (o más bien cíclico) permite tener metáforas que no tienen un punto deíctico que impone una cierta dirección (p. ej. hacia el hablante o a partir de él), ya que de cualquier forma el tiempo siempre regresa a un punto similar al punto de origen.

Asimismo, para expresar la idea del desarrollo temporal se usa el gesto "circular" (rolling gesture, en inglés) presentado en la Fig. 7. El uso de este gesto no es limitado a los mayas yucatecos y es muy frecuente en muchas otras culturas (Kendon 1993; Ladewig 2011). El punto crucial es que para los mayas yucatecos queda como la única forma de expresar visualmente el desarrollo temporal. También, por su forma misma, no implica ninguna direccionalidad, lo que sí es coherente con una idea cíclica del tiempo.

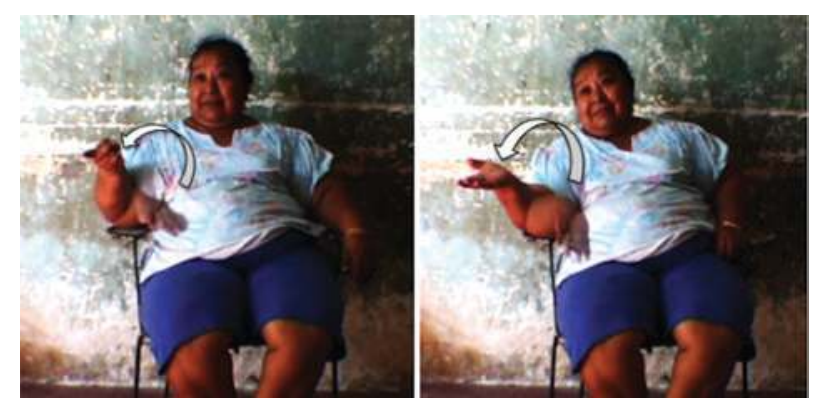

Fig. 7 - Hablante de maya yucateco que produce un gesto similar con sáamal "mañana" (imagen a la izquierda) y jo'olyak "ayer" (imagen a la derecha) [MBC_ent-conv.gestures_2008-08-30].

Tanto en las metáforas verbales como en la representación gestual (es decir visual) del tiempo no existe una línea conceptual en maya yucateco actual. Una prueba adicional viene de un experimento psicolingüístico presentado con más detalles en Le Guen y Pool Balam (2012). En esta tarea experimental diseñada por el proyecto del Max Planck Institute for Psycholinguist con L. Boroditsky (Boroditsky, Gaby, y Levinson 2007) se les pidió a los participantes 
que ordenaran cuatro fichas redondas en las cuales se presenta un evento que se va desarrollando. Por ejemplo, un hombre que va envejeciendo o un plátano que se va comiendo, como se ilustra en la Figura 8.

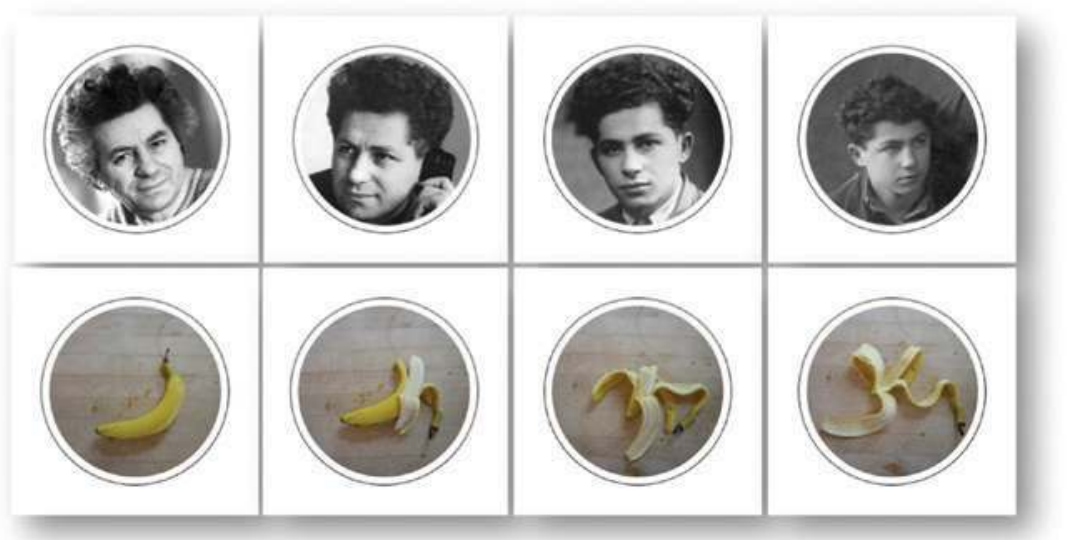

Fig. 8 - Estímulos de la tarea "tiempo en espacio" con 4 fichas que describen un evento que se desarrolla en el tiempo.

La meta de esta tarea es averiguar cómo los participantes "espacializan" el tiempo si el ordenamiento de las fichas revela de manera visual la metáfora temporal que existe tanto en los gestos como al nivel cognitivo. Por ejemplo, para expresar la idea de que el hombre va envejeciendo, en muchas culturas occidentales se usa el sentido de la escritura que va, tal como en español, de la izquierda hacia la derecha; es decir que la imagen del hombre más joven será ubicada más a la izquierda y la del más viejo a la derecha. Sin embargo, en otras culturas, como las semíticas (judía o árabe), como el sentido de lectura de la escritura es a la inversa, pues se ordenarán las fichas a la inversa (es decir, el evento más antiguo se ubicará a la derecha y el evento más reciente a la izquierda). Es de notar que cualquiera de estas estrategias se basan en el punto de vista del hablante. No obstante en ciertas culturas se ordena el tiempo según un marco de referencia geocéntrico. Boroditsky y Gaby (Gaby 2012), por ejemplo, muestran cómo ciertos grupos aborígenes de Australia siempre ordenan las cartas tal como conciben el tiempo: el pasado hacia el oeste y el futuro hacia el este, siguiendo metafóricamente la trayectoria del sol, ver la Figura 3 arriba.

El caso maya yucateco es interesante y original porque no existe una representación en la cual el tiempo se desarrolla en el espacio de forma lineal. Por eso probablemente, una de las estrategias más usadas entre los participantes mayas fue apilar las cartas, así como se presenta en la Figura 9. Para los mayas yucatecos, parece que cada nuevo evento viene a tomar el lugar del anterior, y así los participantes ordenaron las cartas: el más antiguo abajo y el más reciente arriba. 


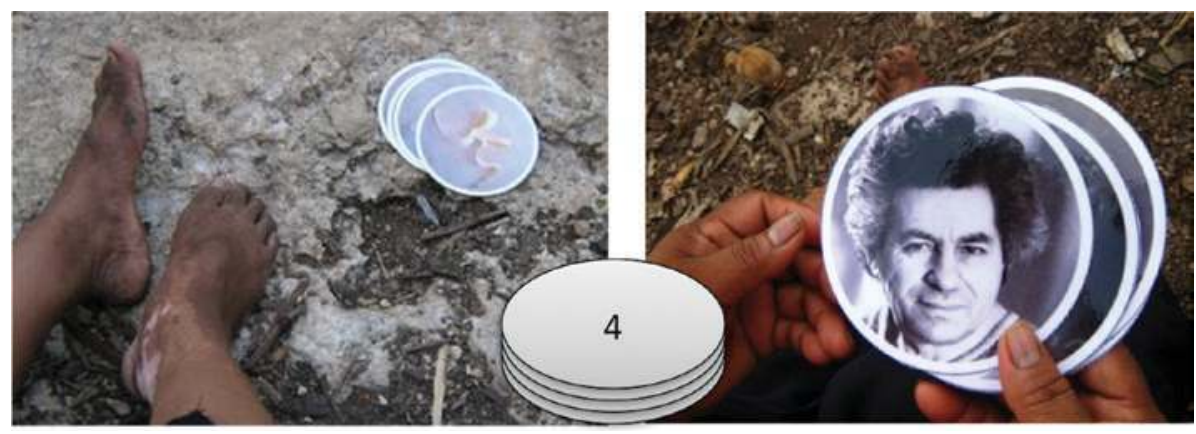

Fig. 9 - Ordenamiento apilado elegido por la mayoría de los participantes maya yucatecos.

Por lo tanto este ordenamiento no implica que exista una metáfora del tiempo desde abajo hacia arriba o viceversa. Esto se comprobó con una segunda tarea presentada en Le Guen y Pool Balam (2012) en la cual los participantes, cuando se les pidió que agregaran un nuevo evento, tomaron el ítem que estaba debajo de la pila para ponerlo encima y así sucesivamente.

En resumen podemos observar dos cosas. La primera es la ausencia de espacialización del tiempo de forma linear. Incluso con el uso de verbos como "ir, regresar, llegar", vemos que los hablantes no imponen un eje o una dirección a su representación del desarrollo del tiempo. El segundo punto es que vemos una variedad de formas de expresar el tiempo. En esta variedad, una constante parece ser que el tiempo "no va a ningún lado" y los eventos se remplazan uno al otro (punto que retomaremos más adelante en los datos coloniales).

\section{La expresión del tiempo en la lengua de señas maya yucateca}

Así como en el caso de otras lenguas de señas del mundo, la misma concepción del tiempo se usa entre la lengua de señas y la(s) lengua(s) oral(es) con la(s) cual(es) está en contacto. De esta forma, no es sorprendente constatar que en la lengua de señas maya yucateca no existe una línea conceptual del tiempo.

Es importante recalcar que es muy común que las metáforas gestuales en uso entre los hablantes sean derivadas de las metáforas orales (así como se mostró en la sección "Metáforas temporales en la gestualidad"). En muchos casos, estas producciones gestuales se transmiten a las lenguas de señas entre los señantes que están en contacto con estas lenguas. Más bien, la producción de las señas y el campo semántico del tiempo derivan directamente de los hábitos gestuales de los hablantes de la comunidad del entorno. Por ejemplo la línea conceptual que existe en las lenguas indoeuropeas se retomó de la misma forma para conceptualizar visualmente el tiempo en muchas lenguas de señas institucionalizadas tales 
como la lengua de señas francesa, inglesa e italiana (Kendon 1993) así como en la lengua de señas americana (o ASL, véase Valli et al. 2000) e incluso en la lengua de señas de Israel (Meir y Sandler 2008, p. 101ff).

\section{Metáforas espaciales y concepción no-lineal del tiempo en LSMY}

En la LSMY - pero también en otras lenguas de señas emergentes del mundo como en la lengua de señas Kata Kolok, usada en Bali (de Vos 2012) o en una lengua de señas secundaria conocida como Walpiri (Kendon 1993)-, los señantes no usan una línea del tiempo para oponer futuro y pasado. La estrategia que utilizan sólo opone presente (ahora) y tiempo distante (o no-ahora) en el cual futuro y pasado están fusionados. Es muy probable, aunque no esté explícitamente mencionado por los autores, que esta estrategia sea usada por los hablantes de las comunidades en las cuales se desarrollan las lenguas de señas ${ }^{12}$.

En la LSMY, la metáfora es la del maya yucateco, es decir que el aquí-ahora está representado por una misma seña con el dedo apuntando a los pies del señante (Figura 10a) y el espacio o tiempo (pasado y futuro) distante por la mano levantada hacia arriba de la cabeza (Figura 10b).
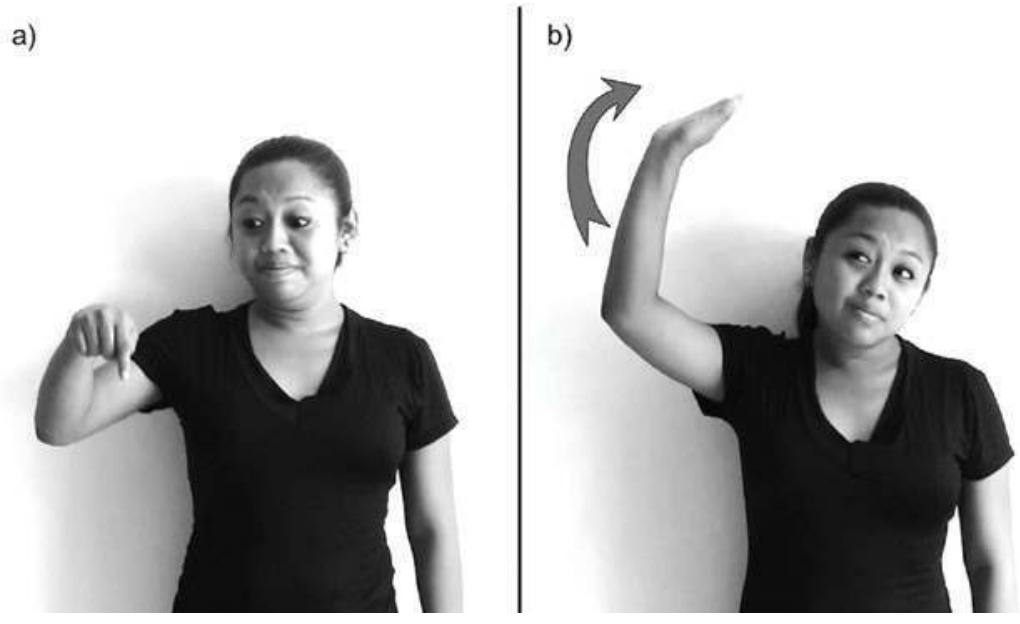

Fig. 10 - Oposición entre (a) AQUÍ-AHORA ${ }^{13}$ y (b) ESPACIO-TIEMPO DISTANTE ${ }^{14}$ en LSMY.

12. Lo es en el caso del Walpiri, Kendon comunicación personal.

13. El video de esta seña se puede consultar en línea siguiendo el enlace: http://ymslproject. org/VIDEOS_datos/waye'.mp4

14. http://ymslproject.org/VIDEOS_datos/\%C3\%BAuchih.mp4 


\section{Estrategias de desambiguación y creación lexical}

Sin embargo, si bien podemos reconocer que estas señas provienen de los gestos co-verbales de los mayas yucatecos, la pregunta que surge es: ¿Cómo se desambigua el pasado del futuro en la LSMY? Es importante recordar que aun cuando los gestos co-verbales para el futuro y el pasado distante son similares, están distinguidos por el habla, es decir que van acompañados por frases como ts'uyúuchtal "hace mucho tiempo (en el pasado)" o yan uyúuchtal "en mucho tiempo (en el futuro)".

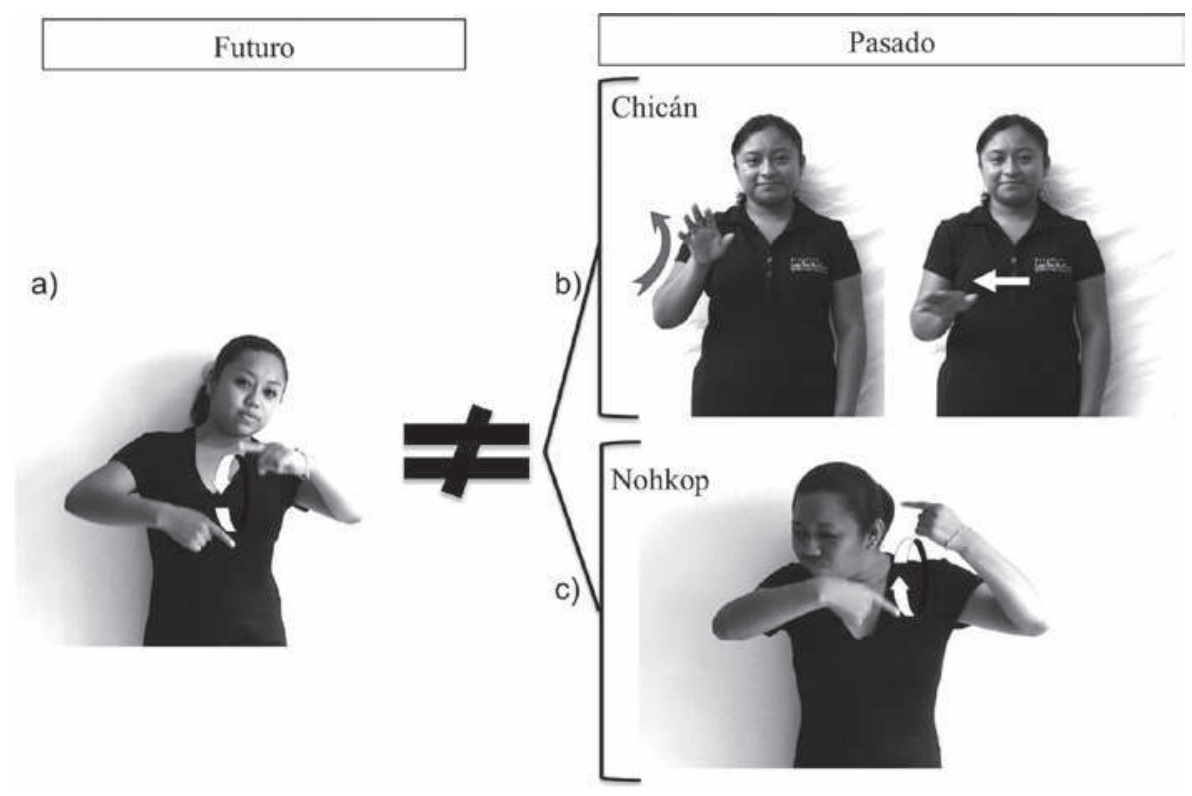

Fig. 11 - Estrategias de creación de señas para el pasado en Chicán y Nohkop. ${ }^{15}$

Para hacer esta distinción existen varias estrategias en LSMY, diferentes según el pueblo. Cabe mencionar que la seña presentada en la Figura 10b, cuando está usada sola tiene el sentido de futuro. Asimismo, tiene un sinónimo que es la seña llamada PASSt (prestada del gesto circular, véase la Figura 7 en la cual se usa una o dos manos girando hacia adelante). Esta seña se usa para hablar del tiempo que pasa y también del futuro. Las innovaciones de la LSMY tienen que ver con la creación de señas para el pasado, y existen dos estrategias según los pueblos en los cuales surgió la LSMY, así como se ilustra en la Figura 11a. En Chicán, se usa la seña para tiempo distante hacia arriba más otra seña de NEGACIÓN en un

15. (a) http://ymslproject.org/VIDEOS_datos/PASSt-yan\%20uy\%C3\%BAuchtal.mp4; (c) http://ymslproject.org/VIDEOS_datos/ts'ok\%20um\%C3\%A1an\%20k'iin.mp4 (nótese que la seña en este ejemplo está intensificada: "muy en el pasado"). 
compuesto (Figura 11b). Nótese que existe una estrategia similar y sinónima en Chicán que es usar la seña de PASSt más la negación. En Nohkop, los señantes en lugar de usar un compuesto tal como en Chicán, decidieron inventar una seña basada en PASSt usándola de manera contrastiva es decir basándose en la iconicidad de la seña pero considerando un flujo temporal inverso (Figura 11c). Es importante precisar que la seña no se hace "hacia atrás" (o sea, el movimiento se desarrolla en el mismo lugar y no hay movimiento según un axis horizontal) y más bien se hace en el sentido contrario de la señas PASSt para desarrollo del tiempo (o futuro), según un movimiento circular invertido.

\section{Formas de ordenar eventos en el tiempo}

En muchas lenguas de señas nacionales (LS francesa, inglesa, de Isreal, ASL, etc.), se pueden visualmente alinear eventos según una línea temporal que origine un poco en frente del cuerpo del señante: icónicamente se representa eventos más antiguos hacia atrás y eventos más recientes hacia delante (Pfau, Steinbach, y Woll 2012, p. 189-190). En LSMY esta estrategia no es posible ya que no existe ninguna línea conceptual del tiempo. En el caso de la LSMY los señantes tienen que hablar de un evento, marcar que se finalizó y hablar del siguiente, etc. En otros términos, esto implica que en una narración, tal como en maya yucateco, un evento tiene que ser concluido (marcado como tal) antes de que otro pueda empezar. En el ejemplo siguiente se presenta un extracto de una enumeración por una señante sorda de Nohkop. Está narrando todas las imágenes que vio en una tarea anterior. Cuando termina de comentar una imagen o un evento, puntúa su enunciado con la seña TS'OK a manera de transición entre los ítems o los eventos, como se presenta en la Figura 12. En este caso, estamos frente a un proceso de desemantización de la seña que adquiere el simple estatus de marcador discursivo (cumpliendo una función equivalente a la de una coma en el lenguaje escrito o de un conector tipo " $y$ " en la lengua hablada). En el análisis que se presenta, se considera que esto es la última etapa del proceso de gramaticalización de una seña que en primer lugar representa un evento como terminado.

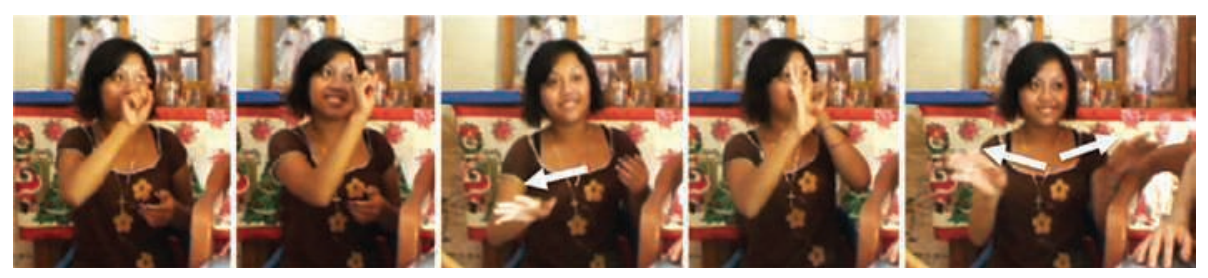

Fig. 12 - Ejemplo de un extracto de una enumeración en LSMY (Nohkop). La señante usa la seña TS'OK para marcar que su descripción está terminada y empieza a hablar de la cosa siguiente. 
La conclusión de esta comparación con el maya yucateco muestra claramente que se transmitió una concepción no-lineal del tiempo a la nueva lengua de señas. Este punto nos permite mostrar dos cosas. La primera es que la metáfora temporal que existe en la lengua hablada (y gestualizada) del entorno influye directamente en cómo se conceptualiza el tiempo en la lengua de señas (así como es el caso de las lenguas de señas en contacto con lenguas indoeuropeos que usan una línea temporal). La segunda, que resulta de la primera, es que usar una línea para expresar el tiempo no es debido a la modalidad visual.

\section{La expresión del tiempo en los libros del chilam balam}

Los textos conocidos como Chilam Balam son de naturaleza profética, lo cual implica dos cosas: una es que son discursos de un género discursivo muy particular, y por lo tanto tiene una forma muy peculiar si se compara con el maya actual y probablemente con el maya usado cotidianamente en aquel tiempo. La segunda implicación tiene que ver con el contenido que era y es aún más ahora, esotérico y remite a profecías. Este último punto significa que tenemos un uso muy importante de formas gramaticales de futuros proféticos que no se usan sino muy raramente en la actualidad (una incluso desapareció).

Es importante mencionar cómo se presentarán los textos del Chilam Balam en esta sección. Los ejemplos provienen de un estudio en curso (Le Guen y Machault, en preparación) y se presentan en cuatro líneas de transcripción y traducción ${ }^{16}$. Los textos son extractos de tres fuentes: del Chilam Balam de

16. La primera es la frase tal como está escrita en el texto original (desde la paleografía). La segunda línea es el texto reescrito usando la ortografía moderna. No se trata de ninguna forma de reescribir el texto en lo que sería el maya moderno, sino de respetar la pronunciación del maya. Es fundamental entender que la ortografía colonial no rinde cuenta de muchos rasgos de la lengua maya, en particular el valor de las vocales (tonos largo, alto, vocal corta y rearticulada) o en ciertos casos las consonantes glotalizadas. Sin embargo, como lo menciona Hanks (2009), ciertos autores coloniales son más sistemáticos que otros en la escritura tal como es el caso de Acuña. Por ejemplo la oración presentada en el ejemplo (extracto del Chumayel) está escrita como en la frase siguiente: tu kinil yulel yx puc yola. De esta oración podemos notar que no se marcan el valor de las vocales. Por ejemplo, kin "sol" se pronuncia $k$ 'iin con tono largo. La construcción yulel es la forma en tercera persona de la raíz $u$ 'ul (rearticulada) "llegar". Finalmente, el nombre de una entidad yx puc yola: está escrito con yolal que es otra vez la marca de tercera persona con la raíz óol (con tono alto) y un sufijo de posesión. Con la ortografía moderna, el ejemplo se puede reconstruir como sigue (las letras en corchetes indican reconstrucciones agregadas): $t\left[i^{\prime}\right] u-k{ }^{\prime} i i n-i l[u]$ y-u'ul-el Yx Pu'uk [u]y-óol-al. La tercera línea propone una interpretación de las palabras y morfemas (es decir la glosa) que permite respaldar la validez de la interpretación (o su crítica constructiva por el lector). Es de mencionar que para este análisis se considera la gramática del maya yucateco colonial y no únicamente la del maya moderno. La cuarta línea es una traducción al español, a veces literal para tratar de restituir el significado o la construcción usada en maya. 
Chumayel (folio 93) ${ }^{17}$, del Tizimin (que se encuentra dos veces en los folios $14 \mathrm{r}^{18}$ y $16 \mathrm{v}^{19}$ ) y del Oxkuztcab (retomada de los cuadernos de Berendt (1868), cuaderno titulado "Lengua Maya miscelánea II", p. 215).

\section{La organización de las profecías}

En las profecías analizadas, aunque podría decirse que para las profecías mencionadas en los Chilam Balam en general, la organización es siempre similar; cada profecía siempre empieza con una fecha del calendario y una mención del lugar en el cual se "asienta" (jeets') el $K$ 'atun (es decir la unidad calendaria de 20 años del calendario tsolk'iin maya yucateco). En el caso de las profecías analizadas, todas son de Hun Ajaw en los tres libros (Tizimin, Oxkutzcab y Chumayel) y siempre el lugar en el cual se asienta el tiempo es Emal, una ciudad costeña del noreste de la península de Yucatán (véanse los ejemplos 13 a 16) ${ }^{20}$. Este tipo de anuncio de la fecha recuerda a las inscripciones glíficas de las estelas mayas prehispánicas. Sin embargo, en contraste con las inscripciones que marcaban la fecha con la cuenta larga, los Chilam Balam solamente cuentan ciclos de tiempo de katunes, o sea periodos de 20 años. En los textos se establece una fecha de inicio del evento y este evento nunca está conectado con otro anterior o posterior (aparte de la fecha indicada) ${ }^{21}$. La idea maya subyacente que se argumenta en este trabajo a partir de los datos modernos, es que cada evento va remplazando al evento anterior.

Al comparar las profecías, vemos dos formas de presentar la fecha ${ }^{22}$. La primera es la del ejemplo (13) del Tizimin (que empieza en el folleto 14r) y del ejemplo (14) de Oxkutzcab que solamente nos indica el asiento del katún. En cada ejemplo, propongo una traducción completa del texto antiguo en cuatro líneas.

17. Profecia por el katún 1 ajaw del Libro de Chilam Balam de Chumayel, Princeton University Digital Library, p. 97-99, consultada el 13 de marzo 2014, http://pudl.princeton. edu/viewer.php?obj=0z708w51x - page/97/mode/1 up (también en Edmonson (1986, p. 208) (cap. 33).

18. Se encuentra en Edmondson (1982, p. 138) (cap. 27).

19. Se encuentra en Edmondson (1982, p. 140) (cap. 28).

20. Esta formulación también es típica de los discursos rituales actuales que siempre empiezan con un índice temporal y espacial para el beneficio de las entidades sobrenaturales a las cuales se dirige el ritual (Hanks 1984; Le Guen 2006).

21. Al parecer, cada evento en los textos rituales (orales o escritos) es independiente y nunca observamos formas de conectarlos (por ejemplo con expresiones de tipo "antes del evento x" o "a consecuencia del evento y"). Este proceso puede confundir a lectores no mayas y tal vez sea por eso que Edmonson (1986) consideró que las crónicas del Chilam Balam de Chumayel eran "desorganizadas".

22. Debido a esta misma organización, podemos distinguir cuando termina una profecía y cuando empieza la siguiente. 
(13) Apertura de la profecía Hun Ahau en le CB de Tizimin (14r)

Emal U hetz' Katun

$\begin{array}{lll}\text { emal } & u \text {-jeets' } & \text { k'atun } \\ \text { Emal } & 3 \text { A-asiento } & \text { k'atun }\end{array}$

Emal es el asiento del katún

Ti hun Ahau

ti' jun ajaw

PREP uno ajaw

En (la fecha de) Hun Ahau

(14) Apertura de la profecía Hun Ahau en el CB de Oxkutzcab

Emal $\mathrm{u}$ heo katun

emal u-jeets' k'atun

Emal 3A-asiento k'atun

Emal es el asiento del katún

Hun Ahau

Jun Ajaw

uno Ajaw

es (a la fecha de) 1 (Hun) Ahau

En contraste, en las formulas del Tizimin (16v), reproducidas en (15) y del Chumayel, reproducidas en (16) se menciona el número del katún con el clasificador numeral $t s$ ' $i$ it usado para cosas alargadas y delgadas (un punto que desarrollo más adelante). En el Tizimin (16v) se menciona que el katún es el sexto cuando en el Chumayel se trata del séptimo.

(15) Apertura de la profecía Hun Ajaw en el CB de Tizimin (16v)

Hun Ahau katun

Jun Ajaw k'atun

uno Ajaw k'atun

katún 1 Ahau

uac sit ti katun

[u-]wak ts'it ti' k'atun

3A-seis NC PREP k'atun

(en el) sexto katún

ich cah emal: uhetz katun:

ich kaaj emal u-jeets' k'atun

PREP pueblo Emal 3A-asiento k'atun

en el pueblo de Emal es el asiento del katún 
(16) Apertura de la profecía Hun Ajaw del Chumayel

Hun Ahau katun

$\begin{array}{lll}\text { Jun } & \text { ajaw } & \text { k'atun } \\ \text { uno } & \text { ajaw } & \text { k'atun }\end{array}$

katún 1 Ahau

u uuc sit katun

u-'uuk ts'iit k'atun

3A-siete NC k'atun

su séptimo katún

emal: uhetz katun:

emal u-jeets' k'atun

Emal 3A-asiento k'atun

Emal es el asiento del katún

En resumen, los ejemplos (13) a (16) manifiestan la estructura repetitiva y ritualizada de las profecías, un punto que se detallará más adelante pero que es importante para el argumento de la ciclicidad de la profecía y de la representación del tiempo.

\section{La noción de ciclo y las inserciones}

La comparación de los cuatro textos revela semejanzas en cuanto al contenido y fragmentos en común. Parece que existe un texto base, tal vez el más antiguo, y textos más recientes con más inserciones. Lo interesante es que el texto base representa una estructura repetitiva y fija que no se cambia sino que se rellena y se extiende.

Otra vez, si retomamos la idea de que cada ciclo es independiente y remplaza al ciclo anterior, es difícil imaginar cómo podrían estar articulados entre sí (con una metáfora espacial por ejemplo). Una estrategia para agregar información sin alterar la estructura del texto cíclico es tomarlo como una secuencia grande en la cual se insertan secuencias más pequeñas de la misma naturaleza. En esta sección, vemos cómo se estructura el texto para cada katún y cómo se hacen las inserciones. Tenemos la suerte de tener varios textos de la misma profecía lo cual nos permite comparar las variaciones tomando un texto "base" (el más corto) y sus variantes ampliadas, y analizar cómo se integraron nuevas partes de texto.

En efecto, si comparamos las diferentes profecías Hun Ahau, es posible suponer que los extractos del Tizimin 14r/16r y del Oxkutzcab son los más antiguos, ya que son los más cortos y exhiben más rasgos de un maya arcaico: se nota un uso de formas más antiguas del futuro profético (el sufijo -om, ver más adelante), y una presencia menor de préstamos. En contraste, la 
profecía Hun Ahau del Chumayel es mucho más larga que las demás (157 vs. $\left.49,53,58^{23}\right)$.

Sin embargo, podemos notar inserciones de nuevos fragmentos (especialmente en el Chumayel, probablemente el más reciente). Las inserciones o transiciones nunca están marcadas de manera especial (lo que complica a veces la lectura para entender la coherencia del texto), y sólo se adjuntan al texto. En muchos casos, la transición está marcada solamente con una preposición $\left(t i^{\prime}\right)$ y no resulta claro si se trata de un cambio de sección o de la continuación de la frase o del párrafo anterior. Esta preposición indica una relación. Si bien puede ser traducida de forma espacial en ciertos casos (p. ej. ti' u'ula'ani' "llegó allá", ti' uyajawlil "en su reinado"), su función como relator -e introductor de complemento oblicuo- es mucho más amplia, y en muchos contextos el significado espacial no es relevante (p. ej. $k$ 'oja'an $t i$ ' paal "está embarazada" (lit. "enferma en relación a un niño"), nojoch ti" "es grande para él"). Por lo tanto, es difícil argumentar que cuando está usada para la inserción de una nueva parte del texto, la preposición $t i$ ' se basa en una metáfora espacial (como "estar a dentro de" por ejemplo, así como podría hacerlo pensar la traducción al español).

Existen algunas formulaciones que son "de cierre", tal como ti' uyajawlil, que literalmente significa "en su reinado" y remite al periodo del katún considerado. En el ejemplo siguiente (Tabla 4) se presentan dos extractos de la profecía Jun Ajaw del Chilam Balam de Tizimin (folio 14r/16r) y del Oxkutzcab respectivamente. Podemos notar que son casi idénticos, y sobre todo que, luego de la secuencia de paralelismos y de la lista de los agentes mencionados, ambos textos se concluyen con la expresión ti uyajawlil.

\begin{tabular}{|l|l|l|}
\hline \multicolumn{1}{|c|}{ Tizimin 14r/16r } & \multicolumn{1}{|c|}{ Oxkutzcab } & \multicolumn{1}{c|}{ Traducción del autor } \\
\hline tu pol peten & tu pol peten & en la cabecera de territorio \\
ich can siho, & ich can ziho & en Ich ka'an sij Jo' (Mérida) \\
ti yan yokol & ti yan yokol & está encima \\
ah tzotz matani, & ah tzootz ah matani & el que pide limosna \\
ah mahan koh & ah mahan koh & el que alquila/presta diente \\
ah mahan tz'am & ah mahan pop & el que tiene prestado el trono \\
ah mahan pop & ah mahan sam & el que tiene prestado el petate \\
ti yahau lil, & ti yahaulil & en su reinado \\
\hline
\end{tabular}

Tabla 4

23. Respectivamente: el Oxkutzcab, el Tizimin 14r f. y 16v 
Sin embargo, si los comparamos con extractos de la profecía Jun Ajaw de otras versiones del Chilam Balam, notamos que dicho cierre de secuencia parece ser opcional. Observamos también que el orden de los paralelismos puede variar (como utuukul que encontramos en la primera línea del extracto en el Chumayel, la quinta del Tizimin [folletos 16v/21v], la séptima en el Oxkutzcab y del Tizimin [folletos 14r/16r]). Igual notamos que la expresión ti (u)yaj miatsili(l) ("en su sabiduría") (probablemente en paralelismo con ti uyajawlil en el Chumayel) actúa también como cierre de secuencia de paralelismo.

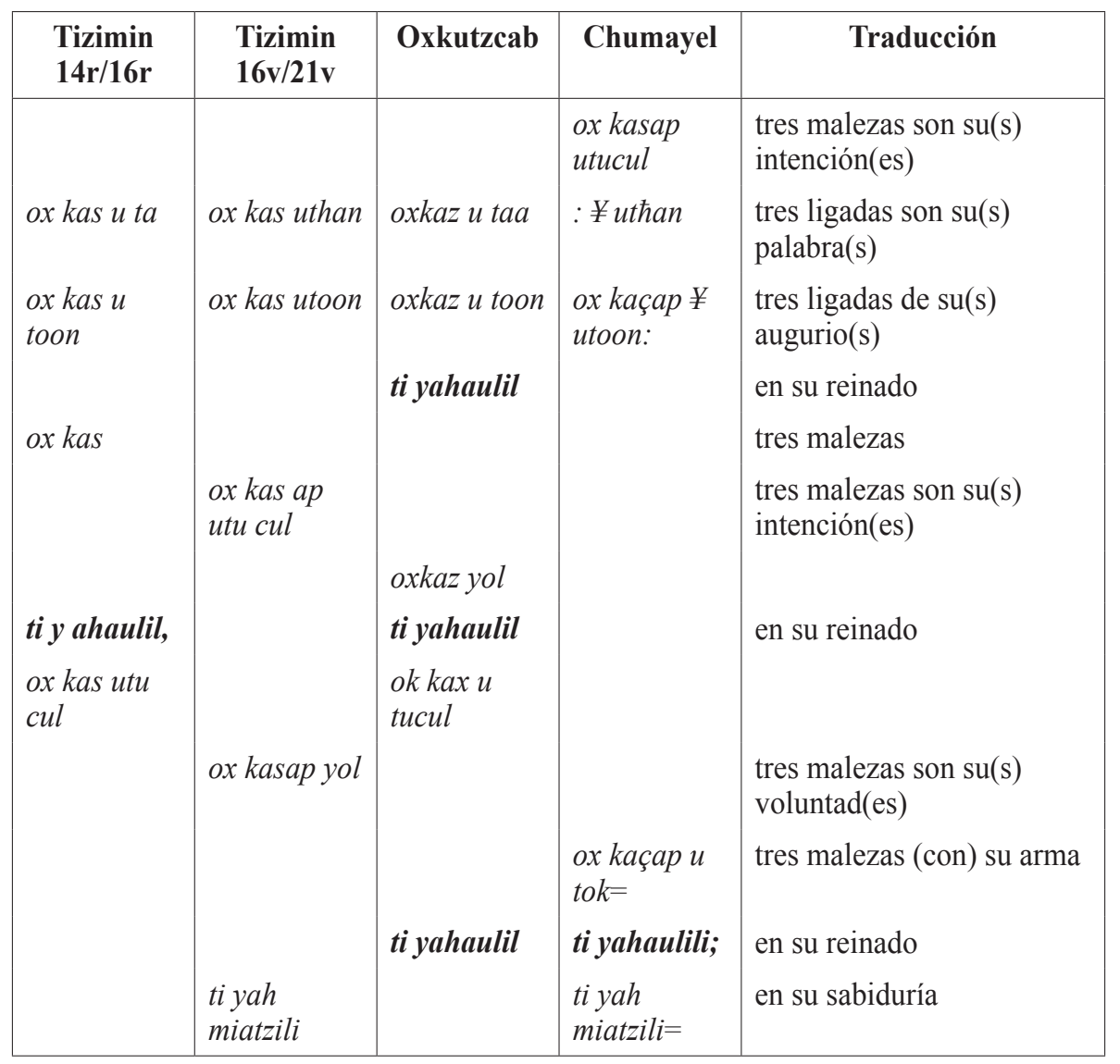

Tabla 5

Más allá, lo que estos ejemplos nos muestran es la existencia de ciclos, muchas veces divididos internamente en paralelismos que se pueden repetir, insertar o completar en la misma profecía. Este proceso es bastante común en ciertos 
géneros narrativos mayas (Vapnarsky 2001) y textos rituales en general (véase entre otros Hanks (1984, 1993), Vapnarsky y Le Guen (2011).

Como lo he mencionado, los textos del Chilam Balam son textos proféticos e históricos, que predicen eventos para cada ciclo de tiempo, implicando unidades temporales cíclicas. La comparación de la misma profecía, repetida a varios ciclos de diferencia (la primera profecía se conoce con fecha de 1640 en el Chumayel, y se repite cada 20 años, aún no está claro cómo fechar las profecías siguientes), revela que aun cuando los textos hablan de eventos diferentes, la estructura del texto y sobre todo la carga (o valencia) que tiene el katún son siempre idénticas (y eso a pesar de su validez histórica y factual). El Hun Ahau es famoso por tener un augurio negativo, lo que se observa en todos los textos considerados. En particular, este katún corresponde a la llegada de los españoles y específicamente de los franciscanos, que el texto menciona mediante la fórmula del ejemplo (17).

(17) El paralelismo que introduce la llegada de la nueva religión cristiana

ti yx yulel

$t i \quad$ ix $\quad[u] y-u$ 'ul-el

PREP CONJ 3A-venir-NOM

llegada también su otra palabra uyanal thani:

$u$-yaanal

3A-diferente

t'aan-i'

palabra- $-\mathrm{TD}_{3}$

uyanal cani:

$u-$ yaanal

3A-diferente

su otra enseñanza kan-i'

aprender- $-\mathrm{TD}_{3}$

La confrontación de los extractos del Tizimin y Oxkutzcab con el Chumayel revela una reinterpretación del mismo katún. Por ejemplo, en la profecía Hun Ahau del Chumayel se menciona al igual la llegada de "una nueva lengua, una nueva enseñanza", sin embargo el texto, que antes trataba de la llegada de los españoles y de la religión católica, está ahora reinterpretado a partir de las aventuras de Andrés Cocom. Se agregaron, según el conteo de Le Guen y Machault (en prep.), 108 reglones en comparación con la profecía más corta del Oxkutzcab. Podemos apreciar la estructura histórica cíclica maya a través de tres capas de interpretaciones del texto. La primera capa es el texto original, o digamos más antiguo, que se refiere a una profecía maya probablemente de origen prehispánico, que resulta bastante obscura en cuanto a su interpretación y a su contenido léxico (se refiere a dioses mayas y a ciertos conceptos posiblemente ya arcaicos en la época colonial). La segunda capa es la reinterpretación del texto maya, pero ahora con la influencia de la ideología cristiana. En este segundo nivel, notamos la influencia franciscana (referencia al Dios cristiano, Jesucristo, la nueva palabra y la nueva enseñanza, etc.) y la presencia del 
maya reducido (Hanks 2009). La última y más reciente capa que encontramos solamente en el Chumayel contiene nuevas inserciones que se refieren a las profecías de Don Andrés Cocom (alias Don Antonio Martínez), presentado como el nuevo Cristo, quien traerá la nueva palabra y la nueva enseñanza (antes presentadas como el hecho de los españoles). Podemos tomar esta última capa como la reinterpretación por los mayas de las dos anteriores, en particular por la asimilación de Don Andrés Cocom al Cristo católico, como figuras mesiánicas. Más detalles sobre esta interpretación se pueden encontrar en Le Guen y Machault (in prep.) y Gunsenheimer (2001).

Si bien no detallaré aquí la interpretación de los varios niveles mencionados, nuestro análisis muestra claramente que los mimos tipos de eventos se repiten de ciclo en ciclo, con cargas equivalentes, ya sea positivas o negativas. Como es obvio en la comparación de los cuatro textos de la profecía Hun Ahau, se trata de periodos diferentes e incluso de eventos diferentes. Sin embargo, y es allí donde podemos ver cómo la concepción maya se revela, a pesar de que se trata de periodos históricos distintos, la concepción cíclica maya trata de relacionarlos para darles una apariencia de repetición. Esta repetición es visible tanto en la valencia positiva o negativa de los ciclos, como también en otros niveles, como en el uso de los paralelismos ${ }^{24}$.

La comparación de estos extractos revela una concepción maya del tiempo, la de una construcción histórica que se repite en el futuro (Roys 1967, p. 91), aun cuando debemos tomar en cuenta que estos textos son intrínsecamente híbridos (así como lo muestran las inserciones en las versiones más recientes).

\section{El uso de marcas de tiempo en las profecías}

En esta sección, se presentan ciertos recursos lingüísticos usados para expresar el tiempo en las profecías del Chilam Balam. Al ser profecías, muchos de los enunciados tienen una forma de futuro, que Vapnarsky (2009) ha considerado para el maya actual ser un "futuro profético o predictivo". Existen dos formas más usadas. La primera, que se usa actualmente es el adverbio temporal bíin, empleado con un verbo en forma de subjuntivo, así como en el ejemplo (18a) extracto del Tizimin $(14 \mathrm{v})$. La segunda forma se presenta a continuación en el mismo extracto $(18 b, c)$ con el sufijo de tercera persona ergativa -om.

\section{(a) bin pecnac peten $i$}

$\begin{array}{lll}\text { bíin } \quad \text { péek- } n-a k & \text { peeten- } i \\ \text { FUT.PROF } & \text { mover.sonar-AP-SBJ } & \text { tierra- } \mathrm{TD}_{3} \\ \text { Se moverá la tierra [la península de Yucatán] } & \end{array}$

24. Remitiendo de hecho a una tradición maya en muchos dominios de la vida cotidiana como ritual (véase Monod-Becquelin, Breton, y Ruz 2010). 
(b) pecnom bal cah $i$

péek-n-om bal-kaaj-i'

mover.sonar-AP-FUT.PROF rodear-pueblo- $\mathrm{TD}_{3}$

Se moverá todo el mundo entero [lit. los alrededores del mundo]

(c) pecnom chumuc cab

$\begin{array}{lll}\text { péek-n-om } & \text { chúumuk } & \text { kaab } \\ \text { mover.sonar-AP-FUT.PROF } & \text { centro } & \text { mundo }\end{array}$

Se moverá el centro de la tierra

Como lo comenta Vapnarsky (2009, p. 277), el uso del sufijo -om está restringido a formula paralelísticas y se usa casi exclusivamente con verbos de movimiento (llegar, venir, entrar, pararse, etc.). Al desaparecer de los textos alrededor del siglo XVIII y al estar ausente del maya yucateco actual, Vapnarsky (2009, p. 273) surgiere que el -om era un arcaísmo incluso en el periodo colonial ${ }^{25}$.

Al lado de las construcciones gramaticales existen ciertos términos (sustantivos) para hablar del tiempo y en particular el término kintzil, o sea con la ortografía práctica $k$ 'iintsil, formado del término k'iin "sol, tiempo, día" y el sufijo absoluto -tsil usado para términos intrínsecamente poseídos (usado en particular para los términos de parentesco, incluyendo en la actualidad los préstamos del español, p. ej. jun-túul maama-tsil "una mamá"). Dado lo que sabemos sobre el término k'iin y el contenido de los textos de los Chilam Balam y las gramáticas coloniales, es muy probable que $k$ 'iin-tsil sea una forma inventada por los franciscanos para crear un término equivalente al español "tiempo". Esta hipótesis tiene varios argumentos. Primero, no existe esta forma sufijada con -tsil en la actualidad, pero debido a que $k$ 'iin en su significado "tiempo, hora, momento" fue remplazado por el préstamo del español oora, esto no es un argumento fuerte. En segundo lugar, $k$ 'iin no es una palabra intrínsecamente poseída, y no requiere el sufijo absolutivo -tsil. Lo que es aún más sospechoso, es el uso poseído con este sufijo (que implica uso no poseído), así como en tuk'iintsil janal "a la hora de comer" que contradice las reglas gramaticales del maya que conocemos. Finalmente, $k$ 'iintsil se encuentra muy poco en los textos escritos en maya, y se menciona mayormente en textos escritos por frailes (véase Hanks 2009, p. 238, 259, 376). Estos argumentos

25. Por ejemplo notamos en el Oxkutzkcab una fórmula similar al ejemplo (18a) con la raíz péek "mover, sonar" pero en vez de la formulación con bíin, la presencia del sufijo -om: pecnom bal cahi

péek-n-om baal-kaaj-i,

la tierra temblará

pecnom petni

péek-n-om pet [e]n-i'

temblará el Petén (la península de Yucatán). 
tienden a indicar que $k$ 'iintsil fue un neologismo de los frailes para traducir el término "tiempo, hora", adoptado luego como préstamo en ciertos textos producidos por los mayas. Lo interesante de este término, sin embargo, es que está formado a partir del término "día" o "sol", que remite al ciclo solar del día, una noción cíclica.

La formación del tiempo gramatical en los textos de la época colonial no ha cambiado mucho respecto a la forma de expresar el tiempo en maya yucateco actual. El uso del futuro profético ya no es tan usual (incluso desapareció el sufijo -om) y aun cuando los Chilam Balam eran también textos proféticos, encontramos con frecuencia el uso del biin (+ subjuntivo) en las gramáticas coloniales. Es posible que haya resultado de la voluntad de los frailes de traducir el futuro del español al maya y no sabemos si se usaba de manera común en el habla cotidiana ${ }^{26}$.

En resumen, vimos que las profecías se basan en ciclos predeterminados (con una valencia establecida), lo cual remite a una concepción del tiempo "estructuralmente cíclico" que hace inteligible por qué el futuro es (hacia cierto punto) predictible entre los mayas.

\section{Metáforas temporales en las profecías}

Aun cuando los textos del Chilam Balam son de carácter un poco especial y por ciertos textos híbridos, resultan ser sin embargo una fuente muy valiosa para explorar las metáforas temporales y averiguar si, efectivamente, ellas no revelan un concepto de línea temporal en el maya yucateco colonial.

Explorando los varios textos de los Chilam Balam (Tizimin y Chumayel) no se encontró ningún término espacial que esté usado con un sentido temporal -como podrían serlo pachal "después" (de paach "espalda") o táanil "antes" (de táan "frente")- que podría remitir a una concepción lineal del tiempo.

Como se presentó en la sección "La organización de las profecías", el tiempo en los Chilam Balam todavía se concibe según el calendario maya formado de katunes (o ciclos de 20 años). En la forma en la cual los textos coloniales nos presentan los katunes, estos están en movimiento constante y se asientan en lugares específicos, por lo general ciudades de la región peninsular. En los textos de Chilam Balam analizados en este trabajo, se usan varios verbos de movimiento para describir el desplazamiento de los katunes: taal "venir", jets" "asentar", kum "asentarse sobre otra cosa", liik' "levantar". Asimismo, encontramos verbos de manera, tales como wuts" "doblar" o nup "juntar cosas iguales". En contraste, notamos que el verbo temporal $t s$ 'ok "terminar" es también muy frecuente en las expresiones temporales mayas como se mostró

26. Sin embargo, Vapnarsky (2009) muestra que el uso del futuro biin en siglos pasados era de uso más amplio que las profecías. 
en las secciones anteriores. Se puede considerar el ejemplo siguiente (19) extracto del Chumayel.

(19) Usoc katun; bay yili ubeltabal tukuchul u uuo katune-

\begin{tabular}{|c|c|c|c|c|}
\hline U-ts'ok & k'atun & bay & {$[u] y-i l-i[l]$} & $\begin{array}{l}u-\text { beel-t-a'ab-al } \\
3 \text { A-hacer-APL-PAS-PART }\end{array}$ \\
\hline $3 \mathrm{~A}-\mathrm{TERM}$ & k'atun & MAF & $3 \mathrm{~A}-\mathrm{ver}-\mathrm{REL}$ & $3 \mathrm{~A}-$ hacer-APL-PAS-PART 3 \\
\hline $\begin{array}{l}t[i]-u-k^{\prime} \imath \\
\text { PREP } 3 \mathrm{~A}-1 \\
\text { (Es el) fin }\end{array}$ & $\begin{array}{l}l l \\
-\mathrm{PART}_{3}\end{array}$ & $\begin{array}{l}u-w u t s \text { ' } \\
\text { 3A-doblar } \\
\text { rató de hace }\end{array}$ & $\begin{array}{l}\text { k'atun-e' } \\
\text { k'atun- } \mathrm{TD}_{4} \\
\text { que llegará el ' }\end{array}$ & blamiento \\
\hline
\end{tabular}

Los términos usados para hablar de los movimientos de los katunes son muy imagísticos pero no parecen estar basados en una visión linear del tiempo sino más bien en la imagen de eventos que se van remplazando. En el caso de los katunes, el nuevo katún toma el lugar del anterior. Esta concepción remite directamente a la concepción actual, tanto en la lengua maya moderna como en las tareas psicolingüísticas (véase en particular la Fig. 9). Es de notar que ningún elemento textual de los Chilam Balam supone una imagen de los katunes en forma de ruedas, así como es común representar el tiempo en la literatura mayista.

Otro aspecto importante es el uso de varios términos para representar los katunes, como si fueran entidades concretas. En los ejemplos (15) y (16), así como en la totalidad de los textos del Tizimin y Chumayel, el uso del clasificador nominal ts 'iit para contarlos revela que se representan lingüísticamente como "cosas alargadas" (ver también el ejemplo [20], extracto de Edmonson 1982, p. 63).

(20) ejemplo con el uso de clasificador ts 'iit para el k'atun

Uuc Ahau Katun

Uuk ajaw k'atun

siete ajaw K'atun

El katún siete Ajaw

U y ox tz'it Katun C u xocol

Uy-óox ts'iit k'atun k-u-xóok-ol

3A-tres NC.cosa.alargada k'atun $\quad \mathrm{HAB}-3 \mathrm{~A}-$-contar.MP-MP

(es) el tercero (de cosas alargadas) katún que se cuenta

También encontramos otra expresión que se relaciona con la medición del katún y podría considerarse como una extensión metafórica. Se trata de la expresión p'is k'atun "la cuenta del katún", que se basa en la raíz p'is "medir", 
usada para hablar de cómo se mide distancias en la milpa. La raíz p 'íis funciona aquí como un clasificador numeral, ya que aparece siempre después de un numeral, informando sobre cuántas medidas son las del katún, así como en el ejemplo (21).

(21) ejemplo con el uso de la raíz p'is como clasificador (Edmonson 1986, p. 101)

Ox kal katun utzcinnabc i

Óox-k'áal k'atun uts-kin-na'ab-(i)k-i'

tres-veinte k'atun bien-TRZER-PAS-FOC?-TD

(cuando) tres ciclos de veinte años de katún terminaron

Ca tac holhun pis katun

$\mathrm{Ka}$ ' tak jol-hun p'is k'atun

CONJ venir.SBJ cinco-diez NC.medir k'atun

(será) cuando vendrá la quincena medición del katún

Siguiendo la misma idea, la raíz chek’ que, según el Cordemex (p. 88), significa "medir a pie o pasos" también se puede aplicar para la medición de los katunes. Aun cuando es difícil explicar precisamente dicha metáfora, es interesante notar otra vez que la metáfora espacial usada para hablar del tiempo, en este caso el "recorrido" del tiempo, no implica linealidad ni direccionalidad.

(22) Ejemplo del uso de la raíz chek' "medir a paso" (Edmonson 1982, p. 37)

Tu kin $\mathrm{Y}$ an u chek be katun

$t-u-k$ 'iin yan u-chek' be' k'atun

PREP-3A-día DEON 3A-medir.a.paso camino k'atun

En el día en que se medirá a pie el camino del K'atun

Si bien podemos pensar que son los humanos quienes recorren el katún caminando - una metáfora basada en el tiempo desde la perspectiva humana-el mismo texto (el Chilam Balam de Tizimin) contiene otra metáfora en la que son ahora los katunes los que "se recorren entre ellos" o "se pisan" (como lo sugiere el uso del recíproco - tambal), o más bien se traslapan. El ejemplo (23) parece así referirse a la transición entre los katunes en el día del primer katún del wiinal o "mes" llamado Póop (con fecha de 1608).

(23) Ejemplo de la raíz chek’ con el recíproco (Edmonson 1982, p. 98)

\section{T u hun te Pop}

$\begin{array}{lll}t-u-j u n & t e & \text { Póop } \\ \text { PREP-3A-uno } & \text { PREP } & \text { petate.nombre.de.mes }\end{array}$

En el primer (día) del (mes) Póop 


\title{
U chek tam ba katun
}

\author{
u-chek'-tamba(l) k'atun \\ 3A-medir.a.paso-RECIP k'atun \\ Se pisan los katunes (entre sí)
}

En el mismo texto, la transición entre katún se puede expresar con formas que no se basan en metáforas espaciales: con el uso de la raíz jel "cambiar" y otra vez el recíproco (-tambal), como en el extracto repetido en (24), o con la raíz k'ex "cambiar, intercambiar" (uk'ex k'atun "el cambio, remplazo del k'atun" en Edmonson (1982, p. 71).

(24) Ejemplo de la raíz hel con el recíproco (Edmonson 1982, p. 101)

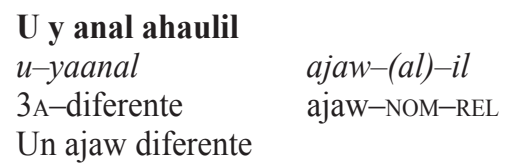

\section{U hel than}

u-jeel t'aan

3A-otro palabra

Otra palabra

\section{U hel ah kin}

$u$-jeel aj k'iin

3A-otro AG sacerdote

Otro sacerdote

\section{T u hel tam ba katun}

$\begin{array}{ll}t-u \text {-jel-tamba(l) } & \text { k'atun } \\ \text { PREP-3A-cambiar-RECIP } & \text { k'atun } \\ \text { Cuando se cambian los katún } & \end{array}$

En resumen, el análisis de los Chilam Balam permite observar el uso de términos concretos, a veces espaciales, para hablar de forma metafórica de la noción abstracta que es el tiempo, al igual que en el maya actual y muchas lenguas y culturas en el mundo. Sin embargo, no hemos encontrado ningún ejemplo en estos textos que se base en una visión de un tiempo que se desarrolla de forma propiamente lineal o donde se imponga alguna orientación precisa. Más bien lo que sobresale es la figura de periodos (los katunes) que se remplazan entre sí, una representación muy cercana a lo que se vio en los datos mayas yucatecos actuales. 


\section{Conclusión: Una concepción maya del tiempo}

El análisis diacrónico de las metáforas temporales mayas yucatecas y de diversos tipos de datos (lingüísticos, gestuales y experimentales), puso a la luz el uso de una concepción no-lineal y cíclica del tiempo, tanto en los textos coloniales como en la actualidad en la lengua hablada y los gestos co-verbales. Identificamos un patrón similar en otra lengua, la lengua de señas maya yucateca que comparte un mismo trasfondo cultural. Específicamente, notamos la ausencia de expresiones espaciales para expresar el tiempo que apuntarían a una forma lineal o a un eje direccional del tiempo.

Lingüísticamente, la expresión básica del tiempo se basa en la referencia al ciclo del sol que, para los mayas, da vueltas alrededor de la tierra, con expresiones construidas con la raíz k'iin "sol, día, tiempo". Otras raíces del maya, tal como sut "dar vuelta" o de préstamos del español, tal como bweelta "vuelta", se usan de manera productiva por los hablantes para describir el desarrollo cíclico del tiempo. La misma idea de revolución está también presente en la gestualidad de los mayas actuales. Estos dato son importantes ya que, según varios autores, la gestualidad es una "ventana hacia la mente", es decir que permite expresar concepciones internas y no verbalizadas.

Aun cuando las construcciones no son exactamente las mismas en la LSMY, la idea de desarrollo del tiempo es similar, expresada con la seña basada en el gesto PASSt que representa el tiempo moviéndose en círculo y eventos remplazándose espacialmente. Analizando las varias metáforas que se usan en el maya tanto colonial como actual, encontramos ciertos verbos de movimiento tales como "ir", "venir", "llegar" para hablar del movimiento del tiempo. Aun cuando en español estos verbos pueden ser usados en metáforas en las cuales el tiempo o el hablante se mueve metafóricamente según un eje, vimos que no es el caso en maya actual y que probablemente tampoco lo fue en el maya colonial, ya que se encuentran también verbos como wuts" "doblar", nup "juntar cosas iguales" (en su uso transitivo, nupik también se refiere a una acción semejante a "doblar"), $k$ 'ex "remplazar" o jel "cambiar" que remiten a la misma representación de ciclos de tiempo que se remplazan.

Aun si las fuentes usadas para el maya colonial son limitadas, es de resaltar que no encontramos ninguna metáfora lineal que refleje una influencia de la cultura española (con expresiones de tipo "antes" o "después", "lejos" o "cerca" en el tiempo, etc.). A pesar del cambio de sistema calendario (de los katunes coloniales al calendario gregoriano) y del contacto con la lengua y cultura española, perduró un sistema cíclico. En Le Guen y Pool Balam (2012) mostramos cómo el ciclo calendario más grande entre los mayas actuales es el año solar. La comparación con las lenguas actuales muestra que esta concepción sigue todavía muy presente (incluso en tareas no-verbales), y nos da argumentos para interpretar fuentes coloniales con más seguridad. 
Este trabajo considera primeramente metáforas lingüísticas, pero nos permite también ir un paso más allá en la interpretación de rasgos culturales. El hecho de que cada ciclo tiene una estructura estable y una valencia idéntica, por lo menos en las profecías del Chilam Balam, permite explicar cómo, tanto los mayas antiguos como los actuales, pueden concebir que el futuro es predecible (ver p. ej. Vapnarsky 2001, 2009).

Finalmente, el que encontramos la misma concepción no-lineal del tiempo en la lengua de señas maya yucateca, y de forma diacrónica (aunque no exactamente similar) en el maya colonial y actual, permite argumentar a favor de una continuidad de la concepción metafórica cíclica del tiempo maya. Podemos proponer que esta concepción se transmitió del maya yucateco a la lengua de señas maya yucateca a través de la comunicación multimodal (en particular la gestualidad), fundamental en la comunicación maya yucateca. Sin embargo, con el avance fuerte del bilingüismo y de la alfabetización así como por el cambio de las actividades tradicionales (p. ej. el abandono progresivo de la milpa a favor de trabajos remunerados en la ciudad), esta metáfora (con su producción gestual) se ve remplazada en la actualidad por la concepción lineal que existe en español, acabando con la representación milenaria maya del tiempo.*

* Manuscrit reçu en décembre 2015, accepté pour publication en octobre 2016.

Este artículo forma parte del número especial Compases y texturas del tiempo entre los mayas: lo dicho, lo escrito, lo vivido.

Agradecimientos - Agradezco a los organizadores de la conferencia "Mesures et Textures du Temps chez les Mayas. le dit, l'écrit, le vécu" como a los editores del $J S A$ y a los dictaminadores por sus valiosos comentarios y críticas que contribuyeron sin duda a mejorar este artículo. También quiero agradecer a mi compañero de sufrimiento en el estudio del maya colonial, Julien Machault y a la dra. Francisca Zalaquett por sus correciones. Mi gratitud va por supuesto y ante todo a los hablantes y señantes de los pueblos mayas de la península (Chicán, Kopchen y Chemax) que siempre han sido tan generosos conmigo al apoyarme en la descripción de sus lenguas y tolerar mis constantes pedidos lingüísticos. Jach Dyoos bo'otik te'ex tujaajil inwóol.

\section{Referencias citadas}

Barrera VÁzquez Alfredo y Silvia Rendón

1948 El libro de los libros de Chilam Balam, Fondo de cultura económica, México D.F.

Bender Andrea, Annelie Rothe-Wulf, Lisa Hüther y Sieghard Beller

2012 "Moving forward in space and time: how strong is the conceptual link between spatial and temporal frames of reference?", Frontiers in Cultural Psychology [on-line], 3, p. 486, https://doi.org/10.3389/fpsyg.2012.00486. 
Blaha Pfeiler Barbara

1996 “Yan difereensia waye’ yéetel máaya yukatáan”, in Ueli Hostettler (ed.), Los Mayas de Quintana Roo. Investigaciones antropológicas recientes, Universität Bern, Institut für Ethnologie, Bern, p. 7-11.

1999 "Identidad y conciencia lingüística en Yucatán”, in Barbara Blaha Pfeiler y Andreas Koechert (eds), Interculturalidad e Identidad Indigena - Preguntas abiertas a la Globalización en México,Verlag für Ethnologie, Hannover, p. 123-133.

Blaha Pfeiler Barbara y Charles Andrew Hofling

2006 “Apuntes sobre la variación dialectal en el maya yucateco”, Península, 1 (1), p. 27-45.

BOHNEMEYER Jürgen

2002 The grammar of time reference in Yukatek Maya, Lincom, Munich.

2009 "Temporal anaphora in a tenseless language", in Wolfgang Klein y Li Ping (eds), Expression of Time, Mouton de Gruyter, Berlin-New-York, p. 83-128.

BoroDITSKY Lera

2000 "Metaphoric Structuring: Understanding time through spatial metaphors", Cognition, 75 (1), p. 1-28.

2001 "Does Language Shape Thought?: Mandarin and English Speakers' Conceptions of Time", Cognitive Psychology, 43 (1), p. 1-22.

Boroditsky Lera y Alice GABY

2010 "Remembrances of Times East. Absolute Spatial Representations of Time in an Australian Aboriginal Community", Psychological Science, 21 (11), p. 1635-1639.

Boroditsky Lera, Alice Gaby y Stephen C. Levinson

2007 "Time in space", in Asifa Majid (ed.), Field Manual, vol. 10, Max Planck Institute for Psycholinguistics, Nijmegen, p. 59-80.

Bracamonte y Sosa Pedro

2010 Tiempo ciclico y vaticinios: Ensayo etnohistorico sobre el pensamiento Maya, Porrula, México.

Brinton Daniel G.

1882 The Maya Chronicles, Library of Aboriginal American Literature, Philadelphia.

BRown Denise F.

1981 Marketing Patterns and Dependence in a Yucatecan Mayan Community, University of Calgary, Department of Anthropology, Calgary.

1994 Yucatec Maya settling, settlement and spatiality, Ph.D, University Microfilms International, Ann Arbor.

BERENDT Carl Hermann

1868 manuscrito no publicado, Biblioteca de la Universidad de Pensylvania.

CALBRIS Geneviève

1990 The Semiotics of French gestures, Indiana University Press, Bloomington.

CASASAnto Daniel y Lera Boroditsky

2008 "Time in the mind: Using space to think about time", Cognition [on-line], 106 (2), p. 579-593, https://doi.org/10.1016/j.cognition.2007.03.004. 
Casasanto Daniel, Olga Fotakopoulou y Lera Boroditsky

2010 "Space and Time in the Child's Mind: Evidence for a Cross-Dimensional Asymmetry", Cognitive Science [on-line], 34 (3), p. 387-405, https://doi. org/10.1111/j.1551-6709.2010.01094.x.

Casasanto Daniel y Kyle Jasmin

2012 "The hands of time: Temporal gestures in English speakers", Cognitive Linguistics, 23 (4), p. 643-674.

COOPERRIDER Kensy y Rafael NúÑEZ

2009 "Across time, across the body Transversal temporal gestures", Gesture, 9 (2), p. 181-206.

Cunill Caroline

2008 "La alfabetización de los mayas yucatecos y sus consecuencias sociales, 1545-1580”, Estudios de Cultura Maya, 31, p. 163-192.

De Ruiter Jan P.

2007 "Postcards from the mind: The relationship between speech, imagistic gesture, and thought", Gesture, 7, p. 21-38.

De Vos Conny L. G.

2012 Sign-Spatiality in Kata Kolok: how a village sign language of Bali inscribes its signing space, Max Planck Institute for Psycholinguistics, Nijmegen, The Netherlands.

EDMONSON Munro S.

1982 The Ancient Future of the Itza: The Book of Chilam Balam of Tizimin, University of Texas Press, USA.

1986 Heaven Born Merida and its Destiny, The Book of Chilam Balam of Chumayel, University of Texas Press, Austin.

Escobedo Delgado Cesar Ernesto

2012 "Chican Sign Language: A sociolinguistic sketch", in Ulrich Zeshan y Connie de Vos (eds), Endangered sign languages in village communities: anthropological GABY Alice and linguisitic insights, Mouton de Gruyter y Ishara Press, Berlin, p. 377-380.

2012 "The Thaayorre think of Time Like They Talk of Space", Frontiers in Psychology [on-line], 3, https://doi.org/10.3389/fpsyg.2012.00300.

GunSENHEIMER Antje

2001 "La historia de Don Andrés Cocom en los Libros del Chilam Balam", Indiana, 17-18, p. 269-288.

HANKS William F.

1984 "Sanctification, structure and experience in a Yucatec Maya ritual event", Journal of American Folklore, 97 (384), p. 131-166.

1993 "The five gourds of memory", in Aurore Becquelin y Antoinette Molinié (eds), Mémoire de la tradition, Société d'Ethnologie, Paris, p. 330-319.

2009 Converting Words. Maya in the age of the cross, University of California Press Los Angeles.

INEGI

2010 III Conteo de población y vivienda 2010. México.

Kendon Adam

1993 "Space, time and gesture", Degrès, 74, p. 3-16. 
LADEwig Silva H.

2011 "Putting the cyclic gesture on a cognitive basis", CogniTextes, 6.

LAKOFF George y Mark JoHnson

1980 Metaphors we Live by, Chicago/London University Press, Chicago and London.

Le Guen Olivier

2006 L'organisation et l'apprentissage de l'espace chez les Mayas Yucatèques du Quintana Roo, Mexique, thèse de doctorat, Université Paris 10, Nanterre.

2012 "An exploration in the domain of time: from Yucatec Maya time gestures to Yucatec Maya Sign Language time signs", in Ulrich Zeshan y Connie de Vos (eds), Endangered sign languages in village communities: anthropological and linguisitic insights, Mouton de Gruyter y Ishara Press, Berlin, p. 209-250.

Le Guen Olivier y Julien Machault

[in prep.] A new translation and interpretation of the Hun Ahau prophecy from the Chilam Balam of Tizimin, Oxkutzcab and Chumayel.

Le Guen Olivier y Lorena Ildefonsa Pool BALAM

2012 "No metaphorical timeline in gesture and cognition among yucatec mayas", Frontiers in Psychology [on-line], 3 (271), https://doi.org/10.3389/ fpsyg.2012.00271.

LEÓN-PorTILLA Miguel

2003 Tiempo y realidad en el pensamiento Maya: ensayo de acercamiento, UNAM, México.

LuxTON Richard N.

1995 The book of Chumayel, the consel book of the Yucatec maya 1539-1638, Aegean Park Press, USA.

MaKemson Maud Worcester

1951 [1 $1^{\text {re }}$ ed.] The Book of the Jaguar Priest: a translation of the Book of Chilam Balam of Tizimin, with commentary, Henry Schuman Inc, New York.

MCNeILl David

1992 Hand and Mind, University of Chicago Press, Chicago.

Mediz Bolio Antonio

1930 Libro de Chilam Balam de Chumayel [en línea], Ediciones del Repertorio Americano, https://www.goodreads.com/book/ show/3057620-libro-de-chilam-balam-de-chumayel

MEIR Irit y Wendy SANDLER

2008 A Language in Space: The Story of Israeli Sign Language, Lawrence Erlbaum Associates, New York.

Monod-Becquelin Aurore, Alain Breton y Mario H. Ruz

2010 Figuras mayas de la diversidad, Universidad Nacional Autónoma de México, México.

NúÑEz Rafael E. y Eve SweETSER

2006 "With the future behind them: Convergent evidence from Aymara language and gesture in the crosslinguistic comparison of spatial construals of time", Cognitive Science, 30 (3), p. 401-450.

Pérez Juan Pio

1949, Códice Pérez, Liga de Acción Social de Yucatán, Mérida, Yucatán, Mexico. 
Pfau Roland, Marcus Steinbach y Bencie Woll (eds)

2012 Sign Language: An International Handbook, Mouton De Gruyter, Berlin, Boston.

REDFIELD Robert y Alfonso Villa Rojas

1934 Chan Kom, a maya village, University of Chicago Press, Chicago et Londres. Roys Ralph L.

1933 The Book of Chilam Balam of Chumayel, Carnegie Institution of Washington, Washington, DC.

1967 The book of Chilam Balam of Chumayel, University of Oklahoma Press, Norman, Oklahoma.

Sinha Chris, Vera da Silva Sinha, Jörg Zinken y Wany Sampaio

2011 "When time is not space: The social and linguistic construction of time intervals and temporal event relations in an Amazonian culture", Language and Cognition, 3 (1), p. 137-169.

TERÁn Silvia y Christian H. Rasmussen

1994 La milpa de los Mayas, la agricultura de los Mayas prehispanicos y actuales en el noreste de Yucatán, DANIDA, Mérida, Yucatan.

Valli Clayton, Ceil Lucas, Kristin J. Mulrooney y Miako Villanueva

$2000 \quad$ [ $3^{\text {rd }}$ ed. $]$ Linguistics of American Sign Language: An Introduction, Gallaudet University Press, Washington, D.C.

VAPNARSKY Valentina

1999 Expressions et conceptions de la temporalité chez les Mayas yucatecos (Mexique), thèse de doctorat, Université de Paris 10, Nanterre.

2001 "Cambio y continuidad en las concepciones históricas mayas: estrategias discursivas y construcción de la memoria colectiva entre los mayas macehuales de Quintana Roo", in Ueli Hostettler y Matthew Restall (eds), Maya Survivalism, Verlag Anton Saurwein, Markt Schwaben.

2009 "Predicción y performatividad en la memoria histórica maya yucateca", in Antje Gunsenheimer, Tsubasa Okoshi Harada y John Chuchiak (eds), Texto y contexto: Perspectivas intraculturales en el análisis de la literatura maya yucateca, BAS, Bonn, p. 257-288.

2016 "No Escape from the Future. Temporal Frames and Prediction in Yucatec Maya”, in Zlatka Guentchéva (ed.), Aspectuality and Temporality: Theoretical and Empirical Issues, John Benjamins, p. 643-678.

2017 "Futuros en contrapunto: proyección, predicción y deseo en maya yucateco", Journal de la Société des américanistes, número especial, ("Compases y texturas del tiempo maya") [en línea]), 2017.

VAPNARSKY Valentina y Olivier Le GUEN

2011 "The guardians of space: understanding ecological and historical relations of the contemporary Yucatec Mayas to their landscape", in Christian Isendahl y Liljefors Persson (eds), Ecology, Power, and Religion in Maya Landscapes, Verlag Anton Saurwein, Germany, p. 191-208.

Villa Rojas Alfonso

1987 Los elegidos de Dios. Etnografía de los Mayas de Quintana Roo, Instituto Indigenista, México. 
